\title{
ABSOLUTE PHYSICAL CALIBRATION IN THE INFRARED
}

\author{
G. H. Rieke ${ }^{1}$, M. Blaylock ${ }^{1,8}$, L. Decin ${ }^{2}$, C. Engelbracht ${ }^{1}$, P. Ogle $^{3}$, E. AvretT ${ }^{4}$, J. Carpenter ${ }^{5}$, R. M. Cutri ${ }^{6}$, L. Armus ${ }^{3}$, \\ K. Gordon ${ }^{1,9}$, R. O. Gray ${ }^{7}$, J. Hinz ${ }^{1}$, K. Su${ }^{1}$, And Christopher N. A. Willmer ${ }^{1}$ \\ ${ }^{1}$ Steward Observatory, University of Arizona, 933 North Cherry Avenue, Tucson, AZ 85721, USA \\ ${ }^{2}$ Department of Physics and Astronomy, Institute for Astronomy, K.U.Leuven, Celestijinenlaan 200B, 3001, Leuven, Belgium \\ ${ }^{3}$ Spitzer Science Center, California Institute of Technology, Pasadena, CA 91125, USA \\ ${ }^{4}$ Harvard-Smithsonian Center for Astrophysics, 60 Garden Street, Cambridge, MA 02138, USA \\ ${ }^{5}$ Department of Astronomy, California Institute of Technology, MC 105-24, Pasadena, CA 91125, USA \\ ${ }^{6}$ Infrared Processing and Analysis Center, California Institute of Technology, Pasadena, CA 91125, USA \\ ${ }^{7}$ Department of Physics and Astronomy, Appalachian State University, Boone, NC 28608, USA \\ Received 2008 January 16; accepted 2008 March 14; published 2008 May 13
}

\begin{abstract}
We determine an absolute calibration for the Multiband Imaging Photometer for Spitzer $24 \mu \mathrm{m}$ band and recommend adjustments to the published calibrations for Two Micron All Sky Survey (2MASS), Infrared Array Camera (IRAC), and IRAS photometry to put them on the same scale. We show that consistent results are obtained by basing the calibration on either an average A0V star spectral energy distribution (SED), or by using the absolutely calibrated SED of the Sun in comparison with solar-type stellar photometry (the solar analog method). After the rejection of a small number of stars with anomalous SEDs (or bad measurements), upper limits of $\sim 1.5 \%$ root mean square (rms) are placed on the intrinsic infrared (IR) SED variations in both A-dwarf and solar-type stars. These types of stars are therefore suitable as general-purpose standard stars in the IR. We provide absolutely calibrated SEDs for a standard zero magnitude A star and for the Sun to allow extending this work to any other IR photometric system. They allow the recommended calibration to be applied from 1 to $25 \mu \mathrm{m}$ with an accuracy of $\sim 2 \%$, and with even higher accuracy at specific wavelengths such as $2.2,10.6$, and $24 \mu \mathrm{m}$, near which there are direct measurements. However, we confirm earlier indications that Vega does not behave as a typical A0V star between the visible and the IR, making it problematic as the defining star for photometric systems. The integration of measurements of the Sun with those of solar-type stars also provides an accurate estimate of the solar SED from 1 through $30 \mu \mathrm{m}$, which we show agrees with theoretical models.
\end{abstract}

Key words: stars: fundamental parameters - Sun: infrared - techniques: photometric

Online-only material: machine readable table

\section{INTRODUCTION}

For many applications of astronomical photometry, an accurate absolute calibration in physical units and at multiple wavelengths is critical. Previous calibrations have made use of two basic approaches (Rieke et al. 1985). In direct calibrations, measurements are made of celestial sources in ways that allow the signals to be compared directly (if sometimes through a long chain of measurements) with signals from calibrated emitters. For indirect calibrations, direct calibrations at wavelengths well removed from those of interest are interpolated or extrapolated through physical modeling of astronomical sources. For direct calibrations, rigorous error analysis is possible, although there is always a risk of systematic terms that are not captured. Error analysis is far more difficult for indirect calibrations, since the systematic errors in theoretical modeling are often not apparent and there is usually no rigorous way to quantify the errors. Therefore, indirect calibrations must be used with caution until they have been confirmed by other indirect approaches or, better, by direct ones.

Infrared (IR) calibrations have often included some aspect of indirect calibration by extrapolating from the high quality visible direct calibrations. There are now high quality direct calibrations in the IR, so the extrapolations can be tested. In this work, we start with the IR calibrations, test their internal consistency, and

\footnotetext{
8 Currently at UC Davis, One Shields Ave., Davis, CA 95616, USA.

9 Currently at Space Telescope Science Institute, 3700 San Martin Drive, Baltimore, MD 21218, USA.
}

then examine the consequences for their extrapolation into the visible. This approach is preferred in principle because stellar behavior is relatively simple in the IR (e.g., small temperature uncertainties have little effect on the shape of a nearly RayleighJeans spectrum for stars of the temperatures considered here).

The $24 \mu \mathrm{m}$ band of the Multiband Imaging Photometer for Spitzer (MIPS) achieves high photometric accuracy-with typical errors that are an order of magnitude smaller than those previously achieved at similar wavelengths (Rieke et al. 2004; Engelbracht et al. 2007). The Infrared Array Camera (IRAC) also provides a large body of accurate and homogeneous photometry in the 3-8 $\mu \mathrm{m}$ range (Fazio et al. 2004; Reach et al. 2005). In addition, a new absolute calibration of unprecedented accuracy is now available in the thermal IR (Price et al. 2004). These advances make it both desirable and feasible to establish a very accurate calibration of the MIPS photometry at this wavelength and to provide guidelines to tie it in consistently with photometry at shorter wavelengths.

Bessell (2005) has reviewed photometry in general, but with only a few comments on the IR. Price (2004) has reviewed IR calibrations. His review concentrates on a huge body of work by Cohen, Walker, Witteborn, Price, and other co-workers on this topic. The review states that the results are "in substantial disagreement with previous direct calibrations," thus leaving open the question of possible undetected systematic errors. In addition, the review points out a number of discrepancies between the measured properties of the Sun 
(e.g., Thuillier et al. 2003) and measurements of solar analog stars, which indicate possible issues in the photometric system.

The availability of large and homogeneous sets of data such as the Hipparcos photometric catalog (Perryman et al. 1997) and the Two Micron All Sky Survey (2MASS) (Cutri et al. 2003) opens new possibilities to probe these issues and to improve calibrations, as well as providing a solid foundation to extend calibrations uniformly over the entire sky. To do so is the goal of this paper. The paper is organized as follows. In Section 2, we review measurements of the absolute flux from Vega at 2.22 and $10.6 \mu \mathrm{m}$, and the extrapolation of these measurements to the MIPS effective wavelength of $23.675 \mu \mathrm{m}$. In Section 3, we discuss an independent approach to calibration using the solar analog method introduced by Johnson (1965a). In Section 4, we show explicitly the discrepancy in the Vega-based visible calibration and the IR one. In Section 5, we recommend adjustments to the absolute calibration of other photometric systems to bring them into agreement with the work reported here. The paper is summarized in Section 6. Those not wishing to plow through the details can go to that section for a summary of the useful results, which include recommendations for an absolute calibration accurate to $2 \%$ or better across the nearand mid-IR.

\section{DIRECT INFRARED CALIBRATIONS OF "VEGA"}

\subsection{Zero Point}

Traditionally, absolute calibration systems have been referred to a "zero point" (ZP) of a magnitude system, usually defined by the spectral energy distributions (SEDs) of A stars. The Johnson/Arizona system defined the ZP as an average of the colors of a number of A stars, not just those of Vega, and as a result the magnitude of Vega is slightly positive $(\sim 0.02)$ at most bands. This situation has caused confusion because some have used Vega by itself to define zero magnitude, introducing a small offset in nominally similar systems. Further complications arise for the wavelengths of interest for this paper because of the contribution of the Vega debris system to the fluxes from this star beyond $10 \mu \mathrm{m}$ (e.g., Aumann et al. 1984). In addition, Vega is a rapidly rotating pole-on star with a significant temperature gradient $(\sim 1500 \mathrm{~K})$ from its pole to its equator (Gulliver et al. 1994; Aufdenberg et al. 2006), so its SED can differ from conventional models that assume a non-rotating star with a single surface temperature.

Nonetheless, there is a large body of data based on Vega. Fortunately, in the IR the differences in photospheric colors due to the rapid rotation and other modeling uncertainties are small (see Price 2004, Figure 5, and Section 4 below). We therefore quote the results relative to the flux density of "Vega," a mythical star with an SED given by a Kurucz 1993 model spectrum of an A0 star (Kurucz 2005) with $T_{\text {eff }}=9550, \log g=3.95$, $\log z=-0.5$, and normalized to measurements of Vega that are corrected, if necessary, for the IR excess from the debris disk around this star. This convention maintains continuity with the large existing body of IR photometry. We have compared this model with the 2003 version (Kurucz 2005); the differences at photometric resolution and at wavelengths longer than $1 \mu \mathrm{m}$ are less than $0.1 \%$, while the $V-K$ color is $0.2 \%$ bluer with the newer model, again a negligible difference for our purposes. Thus, the Kurucz Vega models provide a stable reference baseline in the IR. We provide the specific model we have used in electronic form so it can be utilized explicitly in future work or in adjustments to the calibration (Appendix A). In the following,
Table 1

Absolute Flux Density from "Vega" at 10.6 and $24 \mu \mathrm{m}$

\begin{tabular}{lcc}
\hline \hline Approach & $\begin{array}{c}\text { Flux density } \\
\text { @ } 10.6 \mu \mathrm{m}(\mathrm{Jy})\end{array}$ & $\begin{array}{c}\text { Error } \\
(\mathrm{Jy})\end{array}$ \\
\hline Rieke et al. (1985) & 35.3 & 1.1 \\
$M S X$ weighted average & 35.04 & 0.24 \\
Weighted average of Rieke, $M S X$ & 35.05 & 0.23 \\
Solar analog (this work) & 34.53 & 1.1 \\
All measurements weighted average & 35.03 & 0.23 \\
Average Rieke et al., solar analog & 34.92 & 0.8 \\
Adopted & $\mathbf{3 5 . 0 3}$ & $\mathbf{0 . 3}$ \\
Adopted, F & $9.35 \times 10^{-17} \mathrm{~W} \mathrm{~cm}^{-2} \mu \mathrm{m}^{-1}$ & \\
Hammersley et al. (1998) & 35.2 & $\ldots .11$ \\
Flux Density at $23.675 \mu \mathrm{m}$ & 7.17 & \\
\hline
\end{tabular}

we place "Vega" in quotes because the ZP of the system is defined by an idealized version of this star.

\subsection{Mid-Infrared}

In this subsection, we discuss absolute calibrations near $10 \mu \mathrm{m}$ and use them to establish a "best" value of $35.03 \pm$ $0.30 \mathrm{Jy}$ for the monochromatic flux density of "Vega" at $10.6 \mu \mathrm{m}$. We also show that the absolute measurements at $21 \mu \mathrm{m}$ are consistent with this value and derive a monochromatic flux density for "Vega" at the effective or mean wavelength of the MIPS $24 \mu \mathrm{m}$ band.

For reasons given in the Introduction, we place the highest weight on direct calibrations in the thermal IR. Such measurements are summarized in Table 1. The two most accurate sets of measurements-Rieke et al. (1985) and the Midcourse Space Experiment $(M S X)$ - have estimated errors of 3\% (Rieke et al. 1985 ) or $\sim 0.6 \%$ (Price et al. 2004) near $10 \mu \mathrm{m}$ and are in excellent agreement to within these errors. Rieke et al. (1985) review previous work (Becklin et al. 1973; Low \& Rieke 1974) and show it agrees closely with their calibration, well within the errors of order $7 \%$ quoted for the earlier work. The solar analog calibration in this work agrees to within $\sim 1.5 \%$, as does another calibration conducted in support of the Infrared Space Observatory (ISO) (both to be discussed below). The consistency of these independent determinations indicates that there are no major systematic errors. At $21 \mu \mathrm{m}$, the agreement between the MSX calibration and previous work is also excellent although the errors estimated for the Rieke et al. (1985) measurements are about $7 \%$. Even though Rieke et al. reported a direct measurement at this wavelength, they based the recommended calibration on an extrapolation from the $10.6 \mu \mathrm{m}$ calibration because they felt it was more accurate.

To obtain the best-possible calibration in the mid-IR, we therefore need an optimum way to combine the measurement of Rieke et al. (1985), the three near $10 \mu \mathrm{m}$ from Price et al. (2004), and the solar analog determination from this work. We do so at a wavelength of $10.6 \mu \mathrm{m}$, using the "Vega" SED as the means to interpolate or extrapolate to the same wavelength and thus to relate the measurements to each other. That is, the calibration is relative to the normalization in Price (2004), Figure 5. However, this figure plots the calibration of Rieke et al. (1985) incorrectly. The figure shows the proposed $\mathrm{ZP}$ of the photometric system as if Vega were zero magnitude but Rieke et al. (1985) set Vega at a magnitude of 0.02 . That is, the measurement of Vega should be 0.02 lower than plotted, and hence in even better agreement with the MSX values than indicated (S. D. Price 2006, private communication). 
Table 2

Measurements of the Absolute Flux of "Vega" at $2.22 \mu \mathrm{m}$

\begin{tabular}{lcc}
\hline \hline Reference & $\begin{array}{c}\text { Equivalent 2.22 } \mu \mathrm{m} \\
\text { flux density (Jy) }\end{array}$ & $\begin{array}{c}\text { Error } \\
(\mathrm{Jy})\end{array}$ \\
\hline Walker (1969) & 638 & 64 \\
Blackwell et al. (1983) & 666 & 20 \\
Selby et al. (1983) & 623 & 25 \\
Booth et al. (1989) & 667 & 27 \\
Weighted average & 653 & 13 \\
Corrected for disk & 645 & 15 \\
Extrapolated from 10.6 $\mu \mathrm{m}$ & 649 & 10 \\
"Best" calibration & $\mathbf{6 4 7}$ & $\mathbf{8}$ \\
\hline
\end{tabular}

Various ways to combine the data are indicated in Table 1. For extrapolating to other wavelengths, we define the "monochromatic" flux density to be proportional to the average over a $1 \%$ spectral bandwidth of $v^{-2} \mathrm{f}_{v}=\lambda^{4} \mathrm{f}_{\lambda}$. For example, we determine the MSX value starting from the $N$-band flux density in Tables 1 and 2 of Cohen et al. (1992), extrapolated to $10.6 \mu \mathrm{m}$ according to the "Vega" SED. We used the standard deviation of the biases in bands $A, C$, and $D$ in Table 9 of Price et al. (2004) to estimate a $1.1 \%$ root mean square (rms) scatter and then combined this value with the quoted uncertainties to compute a weighted average of the biases, which was used to adjust the Cohen et al. value for the flux density.

In the following section, we use the Rieke et al. and MSX weighted average as the basis to project the $10.6 \mu \mathrm{m}$ calibration back to $2.22 \mu \mathrm{m}$, for comparison with direct measurements there. In Section 3.3.3, we use the direct calibration at $2.22 \mu \mathrm{m}$, the IRAC $8 \mu \mathrm{m}$ measurements of solar-type stars, and the SED of the Sun to obtain the independent new calibration listed as solar analog (this work).

We also tabulate the calibration of Hammersley et al. (1998), conducted in support of the ISO mission. They used the 1993 Kurucz model of Vega to extrapolate from $K$-band measurements, and quoted a photospheric flux density from this model at $10.47 \mu \mathrm{m}$. We have corrected their value to $10.6 \mu \mathrm{m}$ and reduced it by $1.29 \%$ to correct for the $K$-band excess of Vega (Absil et al. 2006). It then agrees excellently with the other determinations. Hammersley et al. show that their measurements of stars within this system are also in close agreement with previous measurements of the same stars. We have not included this calibration in our average because it is not clear how to evaluate the errors, but they would appear to be similar to those of most of the other entries.

All of the approaches are consistent with the adopted value of $35.03 \mathrm{Jy}$ for the monochromatic flux density of "Vega" at $10.6 \mu \mathrm{m}$. We quote a slightly increased error from the pure weighted average value to allow for any residual systematic errors. A measure of the degree of agreement is that the calibration ignoring the $M S X$ result is accurate to $2 \%$ and agrees with the $M S X$ calibration to within $1 \%$.

We can now compute that the corresponding "Vega" flux density at $23.675 \mu \mathrm{m}$ (the mean wavelength of the MIPS band) is $7.15 \pm 0.11 \mathrm{Jy}$, where we have assigned a $1.5 \%$ error to allow for any issue in propagating the calibration to $24 \mu \mathrm{m}$. $M S X$ also obtained a calibration at $21.3 \mu \mathrm{m}$, which is equivalent to a "Vega" flux density at $23.675 \mu \mathrm{m}$ of $7.19 \pm 0.11 \mathrm{Jy}$, i.e., is fully consistent with our extrapolation from $10.6 \mu \mathrm{m}$. We take the average value of $7.17 \pm 0.11 \mathrm{Jy}=3.835 \times 10^{-18} \mathrm{~W}$ $\mathrm{cm}^{-2} \mu \mathrm{m}^{-1}$ as the "best" estimate; we have not decreased the error bar in the average because the two determinations are not completely independent.

\subsection{Near Infrared}

This subsection addresses tying the absolute measurement at $10.6 \mu \mathrm{m}$ to direct calibrations of Vega near $2 \mu \mathrm{m}$. Since the high-weight calibrations are at 2.20 and $2.25 \mu \mathrm{m}$ (Blackwell et al. 1983; Selby et al. 1983; Booth et al. 1989), we correct all of them to $2.22 \mu \mathrm{m}$; this wavelength has the further advantage that it is well removed from strong spectral absorptions in both A and G stars. Figure 5 of Price (2004) demonstrates that the predictions of A-star models are very similar in spectral shape between 2 and $24 \mu \mathrm{m}$, and we obtain a similar result comparing the 1993 and 2003 Kurucz models for Vega. At shorter wavelengths, there can be slight deviations of models. However, the comparison of calibrations at 2 and $10 \mu \mathrm{m}$ and interpolations within this range should be robust.

The flux density from "Vega" at $2.22 \mu \mathrm{m}$ is predicted to be $649 \mathrm{Jy}$, using the Kurucz model normalized at $10.6 \mu \mathrm{m}$ to the "Rieke plus $M S X$ " value. We assign a $1.5 \%$ error to this estimate to include any issues in propagating it from $10.6 \mu \mathrm{m}$. This value is compared with the direct measurements of Vega by Walker (1969), Selby et al. (1983), Blackwell et al. (1983), and Booth et al. (1989) in Table 2. This list of measurements represents all the absolute calibrations in the literature except those based in some way on extrapolating the Vega spectrum (or, in the case of Campins et al. (1985) that are revised in this work). All the reported measurements in this table have been corrected to a wavelength of $2.22 \mu \mathrm{m}$ according to the Kurucz model spectrum of Vega.

Absil et al. (2006) report interferometric measurements of Vega at $2 \mu \mathrm{m}$ that show it has an excess of $1.29 \% \pm 0.19 \%$ within a field of diameter $2^{\prime \prime}$, presumably due to a hot inner circumstellar disk (see also Ciardi et al. 2001). We have corrected the direct measurements downward by $1.29 \%$ to remove the effects of this disk. It is unlikely that the output of the disk exceeds this value significantly. For example, the excess of $\sim 4 \%$ observed by $M S X$ in bands $C$ and D at 12.1 and $14.7 \mu \mathrm{m}$ (Price et al. 2004, Table 4) and an upper limit to the color temperature for the excess of $2000 \mathrm{~K}$ (compare Absil et al. 2006) predict an excess above the photosphere of $0.9 \%$ at $2.2 \mu \mathrm{m}$. This rough upper limit is very close to the measured excess at this wavelength, i.e., there is no missing flux that might lie outside the $2^{\prime \prime}$ field. Although there are accurate direct measurements of the flux from Vega at other IR wavelengths (e.g., Mountain et al. 1985), the lack of detailed understanding of the behavior of the circumstellar material makes it problematic to interpret these measurements to the level of accuracy that can be achieved at $2 \mu \mathrm{m}$.

Nonetheless, because the extent and other aspects of the correction may be more uncertain than indicated by the nominal error bar, we have assigned an error of one percentage point to the correction. The resulting value is $645 \pm 15 \mathrm{Jy}$ for the directly measured flux density from the Vega photosphere at $2.22 \mu \mathrm{m}$. The agreement with the value extrapolated from $10.6 \mu \mathrm{m}$ is virtually perfect. We combine the two values to determine a "best" calibration.

The central result from this section is captured in Tables 1 and 2. The theoretical SED of "Vega" links the accurate absolute measurements of this pseudo-star at 2.22 and $10.6 \mu \mathrm{m}$ well. Because consistent values are obtained in distinct calibrations with completely different chains of measurements and assumptions, this agreement appears not to be undermined by any plausible systematic errors. The uncertainties in our final derived flux densities for this "star" are less than $1.5 \%$ at both wavelengths. 


\section{AN INDEPENDENT VERIFICATION VIA THE SOLAR ANALOG METHOD}

\subsection{Spectral Energy Distribution of the Sun}

A further test of the "Vega"-based calibration is to show that an approach based on a different stellar type is consistent with it. To generate this new calibration, we use the solar analog method (Johnson 1965a). That is, we take the absolutely calibrated measurements of the Sun and apply them as colors to other solar-type stars. This subsection discusses the various forms of solar SED that we examined.

\subsubsection{Measurements of the Solar SED}

We take the solar SED for 0.2 to $2.4 \mu \mathrm{m}$ from Thuillier et al. (2003) and G. Thuillier et al. (2005, private communication). This work supplants previous work, although it is generally consistent with the earlier measurements as summarized by Labs \& Neckel $(1968,1970)$, to within a few percent.

Vernazza et al. (1976; hereafter VAL) provide a careful, critical assessment of the measurements at longer wavelengths. It is concluded that the data out to $12 \mu \mathrm{m}$ are of high quality. Specifically, Saiedy \& Goody (1959) estimate that their measurement at $11.1 \mu \mathrm{m}$ is accurate to $0.7 \%$, standard error. Saiedy (1960) estimates the standard errors at 8.63 and $12.02 \mu \mathrm{m}$ to be 0.9 and $1.8 \%$, respectively. Beyond that wavelength, the measurement accuracy decreases substantially. VAL made adjustments of $\sim 4 \%$ in some measurements to improve the apparent agreement. The quoted errors are also a few percent.

VAL described these results with a semi-empirical model. ${ }^{10}$ The model and the final adjusted set of measurements from VAL are described well by a functional fit due to Engelke (1992), which we will use to represent the long wavelength observations for the rest of this paper. We assign a 5\% uncertainty to the measurements beyond $12 \mu \mathrm{m}$ as represented by the Engelke (1992) function.

\subsubsection{Models of the Solar SED}

We have compared the measurements with two photospheric models that predict the solar SED. They are described in more detail here.

The model of Holweger \& Müller (1974, hereafter HM74) is based on a local thermal equilibrium (LTE) analysis of solar line observations. The thermal structure of the temperature minimum region and the chromosphere lying above the photosphere is controversial. The solar temperature structure appears to have a minimum of $\sim 4000 \mathrm{~K}$ near a depth of $500 \mathrm{~km}$ in the photosphere, with an overlying $1500 \mathrm{~km}$ thick, $7000 \mathrm{~K}$ plateau in the mechanically heated chromosphere. However, the analysis of carbon monoxide (CO) lines indicates a very cool brightness temperature $(\sim 3700 \mathrm{~K})$ at the extreme edge of the solar disk, where the slanted line of sight probes into the low chromosphere (see Ayres et al. 2006). In a study of the CO fundamental lines, Harris et al. (1987) concluded that the HM74 photospheric model was consistent with the visible continuum center-limb behavior and the properties of the $\mathrm{CO}$ fundamental spectrum. Using visible continuum intensities and center-limb behavior in combination with the $\mathrm{CO}$ center-limb

\footnotetext{
10 Adjustments were made in this model by Maltby et al. (1986) and Fontenla et al. (2006). The 2006 paper tabulates the latest version of Model $\mathrm{C}$ for the average quiet Sun. These changes do not modify the computed IR spectrum significantly.
}

behavior, Ayres et al. (2006) recently re-determined the solar photospheric thermal profile, which also closely resembles the HM74-model structure.

Theoretical IR spectra were calculated using the HM74 model and the TurboSpectrum program described by Plez et al. (1993), and further updated. The program treats the chemical equilibrium for hundreds of molecules with a consistent set of partition functions and dissociation energies. Solar abundances from Anders \& Grevesse (1989) have been assumed, except for the iron abundance, $\varepsilon(\mathrm{Fe})=7.51$, which is in better agreement with the meteoritic value. The continuous opacity sources considered are $\mathrm{H}^{-}, \mathrm{H}, \mathrm{Fe},(\mathrm{H}+\mathrm{H}), \mathrm{H}_{2}^{+}, \mathrm{H}_{2}^{-}, \mathrm{He}$ I, $\mathrm{He}_{f f}, \mathrm{He}^{-}, \mathrm{C}$, $\mathrm{C}_{\mathrm{II}}, \mathrm{C}_{\mathrm{I} f}, \mathrm{C}_{\mathrm{II} f f}, \mathrm{C}^{-}, \mathrm{N}$ I, N II, N $\mathrm{N}^{-}, \mathrm{O}_{\mathrm{I}}, \mathrm{O}$ II, $\mathrm{O}^{-}, \mathrm{CO}^{-}, \mathrm{H}_{2} \mathrm{O}^{-}$, $\mathrm{Mg}$ I, Mg II, Al I, Al II, Si I, Si II, Ca I, Ca II, $\mathrm{H}_{2}(\mathrm{pr}), \mathrm{He}(\mathrm{pr})$, $\mathrm{e}_{\mathrm{sc}}^{-}, \mathrm{H}_{\mathrm{sc}}^{-}, \mathrm{H}_{2 \mathrm{sc}}$, where "pr" stands for "pressure induced" and "sc" for "scattering." The main continuous absorber in the IR is $\mathrm{H}_{f f}^{-}$, for which the absorption coefficients of Bell \& Berrington (1987) were used. For the line opacity, we used the atomic and molecular database created by Decin (2000) and Decin et al. (2003). The main molecules included are $\mathrm{CO}, \mathrm{SiO}, \mathrm{H}_{2} \mathrm{O}, \mathrm{OH}$, $\mathrm{NH}, \mathrm{CH}, \mathrm{CN}$, and HF. A full spectrum from 2 to $200 \mu \mathrm{m}$ was generated at a resolution of $5 \times 10^{-5} \mu \mathrm{m}$.

The Fontenla et al. (2006) model assumes a temperature minimum of $4500 \mathrm{~K}$ and is computed only to a temperature of $5374 \mathrm{~K}$ in the low chromosphere. It has been found that the higher chromospheric layers do not affect the spectrum between 1 and $100 \mu \mathrm{m}$. The densities are computed from hydrostatic equilibrium and charge conservation, and the calculation assumes local thermal equilibrium (non-LTE effects have negligible influence on the IR spectrum). Further details are given by Fontenla et al. (2006).

\subsubsection{Synthetic Solar Colors}

All of the approaches discussed above for describing the measurements or modeling the solar output agree excellently in the 2 to $4 \mu \mathrm{m}$ region. There are modest divergences at longer wavelengths (particularly beyond $10 \mu \mathrm{m}$ ), but still generally within the expected errors.

We will therefore use photometry of solar-type stars to help decide among the possibilities. The first step is to compute synthetic colors for comparison with the stellar photometry. We use the Thuillier et al. (2003) solar spectrum at wavelengths short of $2.4 \mu \mathrm{m}$ and the Engelke function beyond $2.4 \mu \mathrm{m}$ to represent the measurements of the Sun. They join in a consistent manner with no re-normalization, as shown in Figure 1. Synthetic colors are also computed directly for the models. To compute the $K$-band signal for HM74, we continued the model to wavelengths short of $2 \mu \mathrm{m}$ based on the Thuillier et al. measurements. The exact form of this continuation has only a modest influence on the $K$ photometric color.

In the following sections, we will use near-IR magnitudes as defined by the 2MASS system. To determine synthetic colors for the Sun, we took the relative response functions from the 2MASS Web site ${ }^{11}$ (originally from Cohen et al. 2003). The information on the IRAC $8 \mu \mathrm{m}$ band is from the IRAC Data Handbook. The MIPS $24 \mu \mathrm{m}$ relative spectral response is taken from the MIPS Data Handbook. We obtained the response of the $V$ filter from Johnson (1965b) and multiplied it by a function proportional to the wavelength to convert it into a relative response function. We convolved the Kurucz A-star SED and the various models for the Sun with these functions. The relative

\footnotetext{
11 http://www.ipac.caltech.edu/2mass/
} 


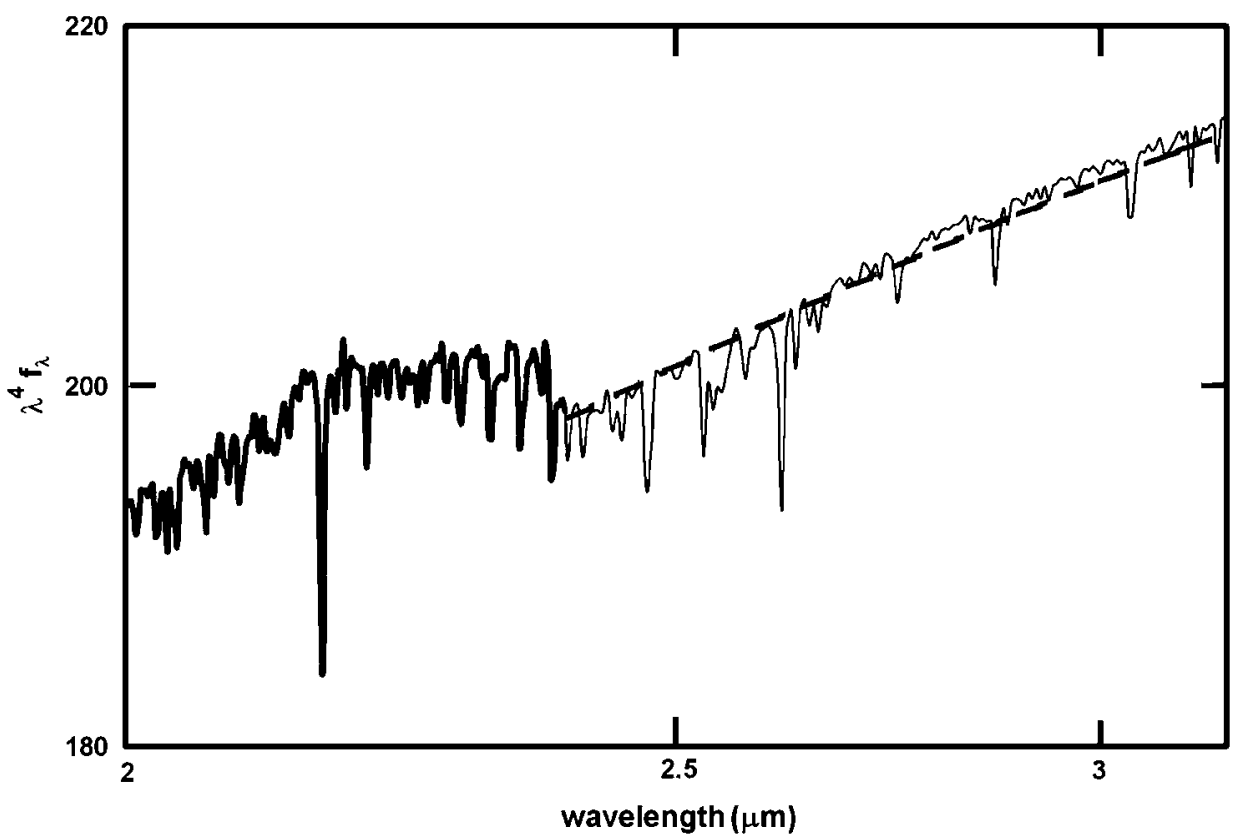

Figure 1. Joining the segments for a complete solar spectrum. The heavy line is the measured spectrum from Thuillier et al. (2003). The dashed heavy line is the Engelke approximation. Both of these curves are left in the measured units with no re-normalization. The light solid line is the modified HM74 model that we use to represent the empirical solar spectrum, normalized to provide a smooth transition from the Thuillier et al. spectrum consistent with the Engelke approximation.

Table 3

Comparison of Solar and Stellar Colors

\begin{tabular}{lcccc}
\hline \hline Band & $\begin{array}{c}\text { Synthetic solar } \\
\text { color (mag) }\end{array}$ & $\begin{array}{c}\text { Observed, } \\
\text { solar-type stars }\end{array}$ & $\begin{array}{c}\text { HM74 } \\
\text { model }\end{array}$ & $\begin{array}{c}\text { Fontenla et al. } \\
\text { (2006) model }\end{array}$ \\
\hline$V_{J}$ & 0.00 & 0.00 & & 0.00 \\
$V-J$ & $1.158 \pm 0.02$ & $1.158 \pm 0.015$ & & 1.20 \\
$V-H$ & $1.513 \pm 0.02$ & $1.484 \pm 0.020$ & & 1.55 \\
$V-K_{S}$ & $1.568 \pm 0.02$ & $1.545 \pm 0.015$ & 1.550 & 1.57 \\
$V-[8]$ & $1.596 \pm 0.02$ & $1.591 \pm 0.015$ & $1.596^{\mathrm{a}}$ & 1.615 \\
$V-[24]$ & $1.54 \pm 0.05$ & $1.590 \pm 0.020$ & 1.577 & 1.564 \\
\hline
\end{tabular}

Notes.

a Adopted value, since the model does not extend below $2 \mu \mathrm{m}$; the Thuillier et al. SED was used to fill in the 1.927-2.00 $\mu \mathrm{m}$ range not included in the model.

responses to the A-star and solar spectra can then be used to calculate the synthetic colors provided in Table 3.

\subsection{Photometry of Solar-Type Stars}

For comparison with the synthetic colors, we determine accurate averages for the measured stellar colors in this subsection. We emphasize the use of large sets of homogeneous measurements (Hipparcos, 2MASS, and homogeneous data sets with MIPS and IRAC). An essential aspect of these comparisons is the linearity of MIPS and IRAC over the range of the observations, which we demonstrate in Appendix B is adequate for our needs.

\subsection{1. $K_{S}-[8]$ Color of Solar-Type Stars and a Solar Analog Calibration}

We first discuss our procedures at $8 \mu \mathrm{m}$ (further details are in Appendix C). The existing calibration of the IRAC photometry is relative to "Vega" as the ZP, as described by Reach et al. (2005). We used their stated ZP to compare their assumed flux density for "Vega" with ours. We first compute the monochromatic flux density at $7.872 \mu \mathrm{m}$, and then apply their recommended color correction of 1.042. Extrapolating from the $2.22 \mu \mathrm{m}$ calibration we find a value $1.2 \%$ brighter than theirs, while extrapolating from $10.6 \mu \mathrm{m}$ we find one that is $1.7 \%$ brighter. Thus, to put their calibration on the same overall scale as is recommended here, an upward adjustment of about $1.5 \%$ is required.

We have used the Formation \& Evolution of Planetary Systems (FEPS) Delivery 3 data products (NASA/IPAC IR Science Archive 2007) for a solar analog calibration at $8 \mu \mathrm{m}$. We also need stars with very homogeneous near-IR photometry. We therefore required that each star have 2MASS measurements of "A" quality in all three bands $(J H K)$, and that they all be measured in the "Read 1" mode (see Appendix D). In addition, we used the Hipparcos photometry at $V$ to compute $V-K$ colors and rejected any star departing by more than 0.10 mag from the standard color. The final sample is listed in Table 8, and the IRAC reductions are described in Appendix C.

To look for intrinsic scatter in the stellar colors, we averaged the 2MASS $J, H$, and $K_{S}$ measurements to a single "Super $K_{S}$ " value (see Appendix C). We have computed the ratio of $2.2 \mu \mathrm{m}$ to $8 \mu \mathrm{m}$ flux densities for the stars in Table 8, using the Super $K_{S}$ magnitudes. We find that the rms scatter is only $2.05 \%$. This value is smaller than would have been predicted from the combination of the uncertainties in the Super $K_{S}$ magnitudes and in the IRAC $8 \mu \mathrm{m}$ flux densities. We conclude that the photometry is well behaved and that all of the stars have very similar SEDs between 2 and $8 \mu \mathrm{m}$. (An exception arises at the $\mathrm{CO}$ fundamental and first overtone bands (band heads at $4.6 \mu \mathrm{m}$ and $2.3 \mu \mathrm{m}$, respectively) due to variations in the absorption strength; these regions are not probed by the photometry we have used for calibration.)

Our empirical solar SED model (Thuillier \& Engelke) lies a factor of 1.026 , or $0.028 \mathrm{mag}$, above the "Vega" SED, at $7.872 \mu \mathrm{m}$, if they are set equal at $2.22 \mu \mathrm{m}$. The average value of $K_{S}-$ [8] from the measurements of the solar-type stars in 
Table 8 is a factor of 0.986 below the Thuillier/Engelke solar SED. The errors in the solar measurement should be small in this region, of order $1 \%$ (VAL). We can derive an independent calibration by normalizing to the results at $2.22 \mu \mathrm{m}$ based on the direct measurements of Vega in Table 2 (i.e., excluding the extrapolation from $10.6 \mu \mathrm{m})$. The resultant calibration is entered in Table 1; we have assigned a $3 \%$ error, based on the error in the $2.22 \mu \mathrm{m}$ calibration and the uncertainties in propagating it to $10.6 \mu \mathrm{m}$. It agrees well with the other calibrations.

We conclude that a completely independent check of the linkage of the 2.22 and $10.6 \mu \mathrm{m}$ calibrations via solar-type stars agrees excellently with the results from direct absolute calibrations and the "Vega" SED at both wavelengths.

\subsubsection{Zero Color at $24 \mu \mathrm{m}$}

We now extend the solar analog method to $24 \mu \mathrm{m}$, to test the various alternatives for the solar SED in this spectral region. We determine zero color at $24 \mu \mathrm{m}$ by averaging the measurements of a large number of A stars, similar in spirit to the original A-star-based ZP (Johnson \& Morgan 1953). In our situation, the approach has a number of virtues. First, because it uses averages of many measurements, it achieves high accuracy in the comparison. Second, peculiar behavior by a few stars will have little influence on the results, and sufficiently peculiar stars stand out and can be rejected as outliers to make their influence disappear entirely. Third, the procedure removes our dependence on previous calibrations.

We selected the sample of stars to use at $24 \mu \mathrm{m}$ from Su et al. (2006) supplemented by stars in the MIPS calibration program. We eliminated all stars with indications of excess emission at either 24 or $70 \mu \mathrm{m}$ (Su et al. 2006 show that $\sim 32 \%$ of typical A stars have excess emission at $24 \mu \mathrm{m}$ ). To guard against subtle excesses, we also eliminated stars younger than $200 \mathrm{Myr}$, since the excesses at $24 \mu \mathrm{m}$ decay roughly as time/150 Myr (Rieke et al. 2005). The stars are listed along with their key parameters in Table 9 (Appendix C). Appendix C also describes our reduction procedures at $24 \mu \mathrm{m}$ in detail.

We have fitted a Gaussian to the distribution of Super $K_{S}$ over $24 \mu \mathrm{m}$ flux-density ratios (normalized to one) for our A-star sample. The standard deviation is 0.048 (we have excluded HD 172728 from the fits because its low values for two measurements imply a possible problem with the 2MASS measurement and also the two stars with the highest ratios, HD 11413 and HD 92845, since they may have weak excess emission). By taking the quadratic difference of the fitted standard deviation and the estimated errors in the $24 \mu \mathrm{m}$ and Super $K_{S}$ values, we find a residual uncertainty term of $3 \%$. This value is an upper limit to the intrinsic star-to-star rms differences in $K_{S}-$ [24] photospheric color.

Because the intrinsic scatter appears to be small, we assume that the scatter in the colors is dominated by measurement errors and it is appropriate to reduce the uncertainties by averaging. We found that attempting to correct the $K_{S}$ measurements for extinction had a negligible effect on the average (0.004 mag) and increased the scatter, so we have used the uncorrected $K_{S}$ values. In an arbitrary normalization that brings the value of the flux-density ratios close to 1 (and will be preserved for a similar calculation for solar-type stars) the average ratio of $K_{S}$ to $24 \mu \mathrm{m}$ flux densities is $0.964 \pm 0.008$.

\subsubsection{4 um Measurements of Solar-Type Stars}

We now apply the identical procedures to $24 \mu \mathrm{m}$ measurements of a suite of solar-type stars. Our sample is drawn largely from the FEPS program, Delivery 3 data products. It is listed in Table 10 and Appendix C gives the details of our reductions. To guard against excess emission, we have only included stars older than 1 Gyr as determined by Wright et al. (2004).

We computed Super $K_{S}$ magnitudes for these stars (see Appendix C). A Gaussian fitted to the resulting distribution of Super $K_{S}$ to $24 \mu \mathrm{m}$ flux ratios (normalized to 1) has a standard deviation of 0.034 . A quadratic subtraction of the estimated measurement errors from the fitted standard deviation leaves less than $1 \%$ for the intrinsic scatter due to variations in the stellar SEDs.

For the calibration calculation, we reject the two lowest and two highest values. The average normalized ratio of $K_{S}$ to $24 \mu \mathrm{m}$ flux density is $1.005 \pm 0.007$. The ratio of the two averages for A and solar-type stars, 1.042, is then the color in flux units of a solar-type star relative to the A-star ZP. It is equivalent to a color in magnitudes of $0.045 \pm 0.011$ in the sense that the solar-type stars are redder than A0V stars. As shown in Table 3 and Figure 2, the resultant value for the Sun at $24 \mu \mathrm{m}$ is $5 \%$ above the Engelke function, and we have assigned an error of $5 \%$ to this function at these wavelengths. Hence, the agreement is within the errors. However, the color of the solar-type stars is well enough determined to suggest that the Engelke function is $3 \%$ to $7 \%$ too blue relative to the true solar SED.

\subsubsection{Solar Analog Calibration at $V, J, H$, and $K$}

We can test the $V-, J-, H$-, and $K$-band calibration by checking to see if we get the correct colors for the Sun. From the synthetic colors, we find $V-K_{S}=1.568$. The error is a combination of that in the $K$ absolute calibration and in the measurements of the Sun. From Table 2, we take the first error category to be $1.2 \%$. The second class of errors is quoted by Thuillier et al. (2003) as 1.1, 0.8, 0.65, and $0.6 \% 1-\sigma$ respectively at $0.95,1.5,1.1$, and $2.5 \mu \mathrm{m}$. We therefore quote a net error of $2 \%$. Similar errors should hold for the other bands.

In principle, this solar color should agree with the colors of similar stars. The $V$ and $K_{S}$ colors of Vega and solar analog stars are tied together by accurate direct calibrations. However, the $J$ and $H$ 2MASS measurements are determined by color transformation and interpolation of the direct calibrations. For 2MASS observations of these relatively bright stars, it is possible that there are residual errors at the $1 \%$ to $2 \%$ level. Therefore, rather than assuming the 2MASS color ZPs, we determined the ZPs for the $J$ and $H$ bands by averaging measurements of a large number of A0V stars also measured in the Read 1 mode. Our procedure is discussed in Appendix D. We then corrected the catalog solar analog colors to these ZPs.

Our sample of solar-type stars is largely from the NStars compilation (Gray 2007). We fitted the trend of colors with temperature and used the fit to adjust them all to match the color expected for a star with a temperature of $5778 \mathrm{~K}$ (the effective temperature of the Sun)-see Appendix D. The final average $V-K_{S}$ color of $1.545 \pm 0.015$ is compared with the $V-K_{S}$ of the Sun in Table 3.

Our value of $\left\langle V-K_{S}\right\rangle=1.545$ for the average of solartype stars differs substantially from standard tabulations such as 1.46 in Tokunaga (2000), as well as other determinations such as that of Holmberg et al. (2006). Part of these discrepancies may be traceable to the 0.045 mag correction implied for the SED anomalies of Vega, but another important contributor is possible discrepancies in translating the solar temperature into the stellar temperature scale (Holmberg et al. 2006). There 


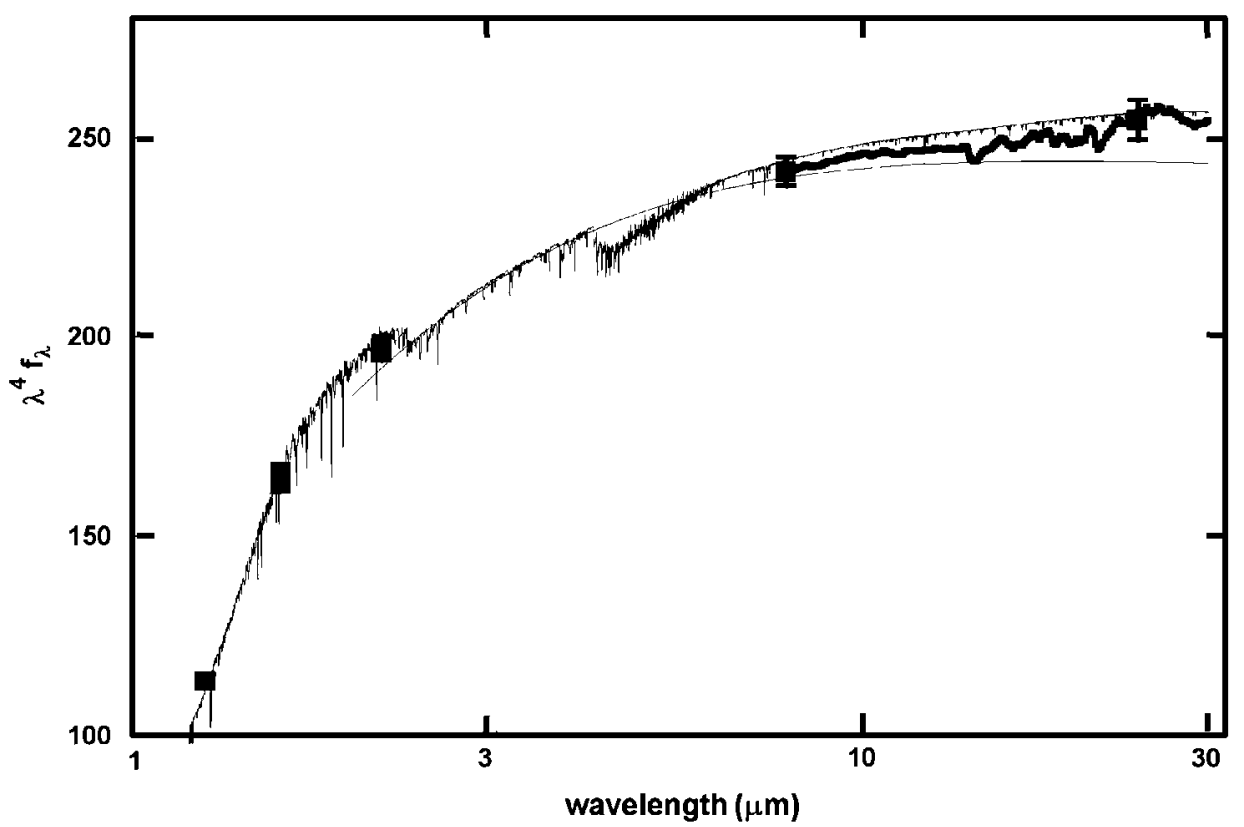

Figure 2. IR spectrum of the Sun. The spectrum has been multiplied by $\lambda^{4}$ to facilitate detailed comparisons in the IR. Out to $2.4 \mu \mathrm{m}$, we plot the measured solar spectrum, based on the results of Thuillier et al. (2003). Beyond this wavelength, the solar spectrum is represented by the HM74 model, normalized and modified at the $\mathrm{CO}$ fundamental bands as described in the text. The photometry of solar-type stars (normalized at $V$ ) is shown as square points, and we have put a $2 \%$ error bar on the measurement at $24 \mu \mathrm{m}$. Between 8 and $32 \mu \mathrm{m}$, we also show the spectrum of solar-type stars obtained with the Spitzer IR Spectrograph (black curve). The smooth curve below the spectrum is the Engelke (1992) approximation to the solar continuum.

Table 4

Solar Analog Colors Corrected to Our System from Bessell et al. (1998)

\begin{tabular}{lcc}
\hline \hline & $V-K_{S}$ & Reference \\
\hline Sun-ref & 1.574 & Colina et al. (1996) \\
Analog & 1.563 & Cayrel de Strobel (1996), Table 6 \\
Model & 1.587 & SUN-OVER $^{\mathrm{a}}$ \\
Model & 1.587 & SUN-NOVER $^{\mathrm{a}}$ \\
& from Table 3 & \\
Solar spectrum & $1.568 \pm 0.02$ & $V, K_{S}$ from Thuillier et al. (2003) \\
Model & 1.57 & Fontenla et al. (2006) \\
ATLAS9 model & 1.556 & Casagrande et al. (2006) \\
Kurucz (2004) & 1.555 & Casagrande et al. (2006) \\
MARCS model & 1.547 & Casagrande et al. (2006) \\
Solar-type stars & $1.545 \pm 0.02$ & This work \\
& NICMOS prime calibrator & \\
P330E & 1.577 & Bohlin et al. (2001) \\
\hline
\end{tabular}

Notes.

${ }^{\text {a }}$ Terminology from Bessell et al. (1998); SUN-OVER refers to ATLAS9 models with overshooting turned on, while SUN-NOVER has it turned off.

is also significant scatter in assigned temperatures within a spectral type in the Nstars compilation (Gray 2007), equivalent to at least one spectral subtype. Roughly speaking, a shift of $10 \mathrm{~K}$ in the temperature of a solar-type star shifts its $V-K$ by 0.01 .

For another comparison, we used a very carefully compiled and clearly described set of colors provided by Bessell et al. (1998): see Appendices A-C of their paper. They define a magnitude system in which $V-K=0$ for Vega, and have forced the calibration to fit both this color definition and a similar model for "Vega" as the one used in this paper. By forcing the "Vega" model to fit the IR calibrations, we find a system in which $V-K=0.045$ for Vega the real star (see below). From Carpenter (2001), we find an additional adjustment of 0.018 from the Glass/SAAO system to 2MASS, or a total of 0.063 . Table 4 allows comparison of the various estimates of $V-K_{S}$ with the Bessell et al. (1998) results corrected to the same basis as ours. Details regarding the first four entries in this table can be found in Bessell et al. (1998). We have also entered the measurements from Table 3 to demonstrate the good agreement. We show additional synthetic colors from models computed by Casagrande et al. (2006). They assumed a $V-K_{S}$ color of 0.047 for Vega, bringing their scale close to the one we have used (with $V-K_{S}=0.045$ ). The prime solar-type NICMOS calibrator, P330E, can be used as an independent test of these colors. We show in Table 4 the colors of P330E relative to a synthetic $V$ magnitude (Bohlin et al. 2001). We have interpolated to provide a $K_{S}$ magnitude. All of these determinations of the solar $V-K_{S}$ color agree very well. 
We also computed $J-K_{S}$ and $H-K_{S}$ colors for the 32 solar-type stars listed in Table 12 with A grade Read 1 measurements in all three bands (this criterion eliminates HD 41330, 90508, 168009, and 186427 from the sample). Since these colors are relative to the 2MASS system, we corrected them for the slight deviations from zero color we found for A0 stars (see Appendix D). The average colors are listed in Table 3 and plotted in Figure 2 for comparison with the solar SED. The errors are estimates from those quoted in the 2MASS catalog, with some allowance for systematic effects. The agreement with the solar colors is excellent.

The IR colors also agree well with those in Tokunaga (2000): $H-K=0.061$ for us, versus 0.05 , and $J-H=0.326$ versus 0.32. We can also compare with Bessell et al. (1998), but we first transform the $J-K_{S}$ to the Johnson/Glass system by subtracting $0.007 \mathrm{mag}$, determined from the Thuillier solar SED. We then find $J-K=0.38$ and $H-K=0.054$, compared with $J-K=0.38$ and $H-K=0.045$ from their tabulation of the solar analog determinations of Cayrel de Strobel (1996).

If we predict errors from the quoted uncertainties in the 2MASS measurements, we obtain a predicted error for a typical $J-K_{S}$ measurement of $3.1 \%$, whereas the scatter in this color indicates a typical error of $2.3 \%$. This behavior is consistent with some degree of correlation in the 2MASS measurements, which is reasonable. There is no indication of scatter in the intrinsic colors of the stars. A similar argument indicates no detected intrinsic scatter in $H-K_{S}$.

We have made a more demanding test for the uniformity of the $J H K$ colors of solar-type stars. We used the accurate near-IR photometry of Kidger \& Martín-Luis (2003), which for well-measured stars has errors of less than $1 \%$. We did not use other available high-accuracy photometry compilations that concentrate on faint sources to calibrate IR arrays, since these stars are more distant and subject to reddening. We selected the 16 stars from Kidger \& Martín-Luis with listed spectral types of G0 through G5 IV or V, with $1.4<V-K<1.8$, with six or more measurements, and with errors in all three bands indicated as $<1 \%$. We then fitted a straight line to the trends of $J-H$ and $H-K$ versus $V-K$, finding scatter around the line of $1.5 \%$ in the first case and $0.9 \%$ in the second. We used $V-K$ instead of spectral type because we did not want the results to be subject to type errors. In addition, Kidger \& Martín-Luis (2003) include a number of color-color plots that show small scatter that is independent of spectral type from A through $\mathrm{K}$ stars. We conclude that $\mathrm{G}$ stars have intrinsic scatter in the near-IR colors of no more than about $1 \%$.

The "anomalously red" color of the solar spectrum as measured by Thuillier et al. (2003) relative to such determinations as Holmberg et al. (2006) has not been satisfactorily explained previously (see discussion in Casagrande et al. 2006). It is comforting that, with care in analyzing the photometric database, we have found that this color is consistent with those of other solar-type stars, and that the scatter in color among such stars is small. As shown in Table 3, this color is consistent not only with the empirically measured solar colors but also with the predictions of a large number of models of the solar SED.

\subsubsection{Spectra}

We have used the Spitzer Infrared Spectrograph (IRS) to confirm the slope and overall spectral behavior of the solartype stellar SED between 8 and $30 \mu \mathrm{m}$. The result, reduced as described in Appendix C, is plotted in Figure 2, normalized to the photometric point at $8 \mu \mathrm{m}$. If it is normalized to the HM74 model at $8 \mu \mathrm{m}$, the rms noise around the model continuum is $\sim 0.7 \%$. It therefore confirms to high accuracy the overall shape of the SED of solar-type stars as described by this model.

\subsubsection{An Empirical Solar SED}

As shown in Figure 2, the HM74 model agrees well with the measurements of both the Sun and of solar-type stars: (1) it tracks the Engelke function closely out to about $10 \mu \mathrm{m}$; (2) it is consistent with the solar-type stellar calibration at $8 \mu \mathrm{m}$; (3) it is also consistent with the solar-type stellar color at $24 \mu \mathrm{m}$; and (4) it agrees with the overall spectral shape of solar-type stars measured with IRS between 8 and $30 \mu \mathrm{m}$. The model also includes a full treatment of the solar absorption line spectrum.

We therefore adopt it to describe the mid-IR SED of the Sun. To normalize it to the Thuillier et al. spectrum, we took advantage of the fact that the absolute level in the Engelke function agrees very closely with the Thuillier spectrum at 2.3$2.4 \mu \mathrm{m}$, so we can use this fit as a smoothing function to help join the two spectra. Figure 1 illustrates how the HM74 model was joined to the Thuillier measurements.

The remaining issue is that the $\mathrm{CO}$ fundamental bands are difficult to fit a priori in models. We empirically adjusted the depth of these features by setting the CO absorption features to be consistent with the spectroscopy of Wallace \& Livingston (2003). At their high spectral resolution, there are a number of atmospheric mini-windows that allow accurate measurement of $\mathrm{CO}$ equivalent widths. On average, we found that the HM74 model had slightly weaker $\mathrm{CO}$ absorption than found by Wallace \& Livingston. We therefore used the Engelke approximation between 4 and $6.5 \mu \mathrm{m}$ as a featureless continuum, normalized to the HM74 model. We multiplied the Engelke SED by 0.125 , subtracted it from the HM74 model, and renormalized the result to the original continuum level to bring the $\mathrm{CO}$ equivalent widths into agreement with those of Wallace \& Livingston (2003). This modified spectrum was used to replace the HM74 values between 4 and $6.5 \mu \mathrm{m}$.

Our final adopted solar spectrum is shown in Figure 2 and given numerically in Appendix A. The Engelke (1992) fit to the VAL model/reconciled measurements falls slightly below the empirical model at wavelengths longer than about $6 \mu \mathrm{m}$. In the IRAC $8 \mu \mathrm{m}$ band, the discrepancy is $1.4 \%$, at the outer limits of the errors. The model is therefore slightly discrepant with our calibration, based on accurate solar measurements. At $24 \mu \mathrm{m}$, the model and the photometry differ from the solar measurements by $5 \%$, but here the model result is to be preferred because of the larger errors in the solar measurements. In general, the model should represent the true solar SED to within $\sim 2 \%$, an error estimated from a combination of the discrepancies with the solar measurements and the photometric errors in the solar-type stellar colors.

\section{VEGA AT WAVELENGTHS SHORT OF $2 \mu \mathrm{m}$}

To extend these procedures to the visible range, we use the average of our "best" calibrations at $10.6 \mu \mathrm{m}$ from Table 1 and at $2.22 \mu \mathrm{m}$ from Table 2 and the "Vega" model to predict a value of $3714 \mathrm{Jy}$ for Vega at $0.5556 \mu \mathrm{m}$. Mégessier (1995) has summarized and reconciled various direct measurements of Vega, corrected to a wavelength of $0.5556 \mu \mathrm{m}$. The preferred value for the reconciled measurements is $3563 \mathrm{Jy}, 4.2 \%$ or $0.045 \mathrm{mag}$ less than we find at this same wavelength via the model. The net errors are only about $1 \%$ for the IR and $0.7 \%$ at $0.5556 \mu \mathrm{m}$. This value agrees with the results of Bohlin \& 
Gilliland (2004), who find that the spectrum of Vega normalized at $0.5556 \mu \mathrm{m}$ and extrapolated using the 1993 Kurucz model to $2 \mu \mathrm{m}$ is $2 \%$ fainter than the calibration of Cohen et al. (2003), which we find in turn is $2 \%$ lower than our calibration. Thus, although the theoretical "Vega" spectrum gives good agreement with measurements in the IR, there is a significant discrepancy between the IR and visible. This result is not new. Mégessier (1995) discusses it at length, summarizing many results that point to the same issue. As a result, this work considers the IR calibration separately from the visible one for similar reasons as discussed here.

It seems likely that the departure from the model arises because Vega is a pole-on rapid rotator. With a measurement of the surface temperature distribution on the star (Aufdenberg et al. 2006), we can now address where its SED might depart sufficiently from the single-temperature models to be of concern for its use as a calibrator. The equatorial surface temperature is estimated to be $7900 \mathrm{~K}$, corresponding to type A7, which has $V-K=0.5, J-K=0.09$, and $H-K=0.03$ (Tokunaga 2000). If we imagine the effective visible surface of the star to be a combination of A0 and A7 spectral type to give a net $V-K=0.045$, by interpolation we expect an effect of $\sim 0.01 \mathrm{mag}$ in $J-K$ and $0.003 \mathrm{mag}$ in $H-K$ to allow for the cooler portion of the surface (these values have little dependence on the exact spectral types used to fit Vega). We conclude that this effect can be ignored in using Vega as a relative IR calibrator at $H$ band and longer wavelengths, but that measurable effects are expected at wavelengths short of $J$.

In Appendix D, we determine a 2MASS $K$ magnitude for Vega of $-0.036 \pm 0.010$ by transforming the measurements of Johnson et al. (1966) into the 2MASS system. Typical adopted values for the $V$ magnitude of Vega are 0.03 (Johnson et al. 1966; Gray 1998) or 0.026 (Bohlin \& Gilliland 2004). The observed $V-K$ of this star is therefore 0.062 to $0.066 \pm$ 0.012 . By comparing the absolute calibrations at $\mathrm{V}$ and in the IR, we found $V-K=0.045 \pm 0.013$. An additional $0.014 \pm$ 0.002 mag should be added to account for the contribution of the ring, for a net $V-K=0.059 \pm 0.013$. The difference in these estimates is 0.003 to $0.007 \pm 0.018$, that is, it is not significantly different from zero. This desirable outcome would appear in part to result from the indirect procedures used to set the $\mathrm{ZP}$ for most photometry since that of Johnson. By setting the $V-K$ colors of a large suite of A0V stars to zero, the systems have been forced to remove any residual anomalies due to the behavior of Vega. The result confirms our derivation of A-star and solar-type colors in this paper under the assumption that there are no unexpected offsets between the visible and near-IR, despite the unexpected behavior of Vega as the star defining the ZPs.

\section{A CONSISTENT CALIBRATION}

We have demonstrated that an absolute calibration can be derived between 1 and $25 \mu \mathrm{m}$ that is consistent with all the direct calibration measurements, and both with A-star standards and with the solar spectrum as reflected by solar-type stars. However, practical photometry is conducted through filters of some band width, which must be taken into account in applying this calibration. To apply any calibration conveniently requires further simplification of its description through definition of a wavelength associated with a measurement and of an equivalent monochromatic flux density at that wavelength, derived from the calibration. There are a number of possible wavelength
Table 5

Multiplicative Factors to Reconcile Mid-IR Calibrations to Proposed One

\begin{tabular}{lcc}
\hline \hline 2 MASS $K_{S}$ & IRAC $7.872 \mu \mathrm{m}$ & IRAS $12 \mu \mathrm{m}$ \\
\hline $1.02^{\mathrm{a}}$ & $1.015^{\mathrm{b}}$ & 0.992 \\
\hline Notes. & \\
a Relative to calibration of Cohen et al. (2003). \\
b Relative to calibration of Reach et al. (2005).
\end{tabular}

definitions. The simplest is the mean wavelength (which we also term the effective wavelength (H. L. Johnson 1972, private communication)). The "nominal" and "isophotal" wavelengths are alternative ways to describe a photometric band. Refer to Appendix E for further discussion of these issues.

The correction factors to put various sources of IR photometry on the same calibration as derived in this paper are listed in Table 5. The existing calibrations are to be multiplied by these factors; for example, the IRAC calibration is slightly faint relative to the MIPS one and flux densities under it need to be increased by $1.5 \%$. Since this discrepancy can be traced to the flux-density estimate for Vega, it should hold for all the IRAC bands. At $10 \mu \mathrm{m}$, the new calibration is $0.8 \%$ lower than the calibration of Rieke et al. (1985) (see Table 1). Since the IRAS $12 \mu \mathrm{m}$ calibration is derived directly from that of Rieke et al. (1985), a similar difference should hold for it (see Beichman et al. 1988). Cohen et al. (1992) re-calibrated IRAS at $12 \mu \mathrm{m}$, finding a value $2.4 \%$ below the Beichman et al. (1988) calibration, and thus $1.6 \%$ lower than the preferred value based on Table 1. Our calibration is $2 \%$ lower than the IRAS one at $25 \mu \mathrm{m}$ (see Beichman et al. 1988). Cohen et al. (1992) also recalibrated this band, finding a value $6 \%$ below that of Beichman et al. (1988) and 4\% below our preferred value.

With careful specification of the defining wavelengths (see Appendix E), we can now compare the various calibrations in the near-IR. The 2MASS calibration at $K_{S}$ by Cohen et al. (2003) is $2 \%$ lower than ours (i.e., the fluxes in the 2MASS system must be increased by $2 \%$ for consistency with our calibration). Appropriate calibration parameters for 2MASS are listed in Table 6 . In addition to the relevant wavelengths and flux densities, the table includes a color correction to a $9550 \mathrm{~K}$ black body to give an idea of the size of such terms for hot stars. The tabulated number is the factor by which the defining SED (flat for a mean wavelength or rising in proportion to wavelength for a nominal one) must be increased relative to the stellar ZP to give the same signal. For objects cool enough that the photometric bands are on the Wien side of their SEDs, the corrections are substantially larger.

The calibration proposed by Tokunaga \& Vacca (2005) for the Mauna Kea Observatories near-IR filter set is $2.6 \%$ lower than ours at $2.22 \mu \mathrm{m}$. The measurement of Vega by Campins et al. (1985) is $1.4 \%$ higher. The homogenized photometry proposed by Bessell et al. (1998) is $3.7 \%=0.039$ mag lower than our proposed calibration at $2.22 \mu \mathrm{m}$. This shift is very likely associated with the red color of $V-K_{S}=0.045$ for Vega. Bohlin \& Gilliland (2004) suggest using the Kurucz Vega model $(T=9550, \log g=3.95)$ to extrapolate from the $V$ calibration into the IR; we have shown that the resulting calibration will be 0.045 mag lower than the direct IR measurements.

\section{SUMMARY}

We have reviewed the calibration of IR (1 to $25 \mu \mathrm{m})$ photometry. Our most important conclusion is that there is 
Table 6

Suggested Calibration of 2MASS Photometry

\begin{tabular}{|c|c|c|c|}
\hline Band & $J$ & & \\
\hline Band width & $0.1625 \mu \mathrm{m}$ & & \\
\hline Average $\mathrm{ZP}\left(\mathrm{W} \mathrm{cm}^{-2} \mu \mathrm{m}^{-1}\right)$ & $3.21 \times 10^{-13}$ & & \\
\hline Wavelength type & $\lambda_{0}(\mu \mathrm{m})$ & $\lambda_{0}^{\prime}(\mu \mathrm{m})$ & $\lambda_{\text {iso }}(\mu \mathrm{m})$ \\
\hline Wavelength & 1.2410 & 1.2444 & 1.2356 \\
\hline $\mathrm{ZP} \mathrm{F} \lambda$ at $\lambda_{0}\left(\mathrm{~W} \mathrm{~cm}^{-2} \mu \mathrm{m}^{-1}\right)$ & $3.16 \times 10^{-13}$ & $3.13 \times 10^{-13}$ & $3.21 \times 10^{-13}$ \\
\hline $\mathrm{ZP} \mathrm{F}_{v}$ at fiducial $\lambda(\mathrm{Jy})$ & 1623 & 1617 & 1635 \\
\hline Color correction/9550 K black body & 1.017 & 1.028 & \\
\hline Band & $H$ & & \\
\hline Band width & $0.2508 \mu \mathrm{m}$ & & \\
\hline Average $\mathrm{ZP}\left(\mathrm{W} \mathrm{cm}{ }^{-2} \mu \mathrm{m}^{-1}\right)$ & $1.164 \times 10^{-13}$ & & \\
\hline Wavelength type & $\lambda_{0}(\mu \mathrm{m})$ & $\lambda_{0}^{\prime}(\mu \mathrm{m})$ & $\lambda_{\text {iso }}(\mu \mathrm{m})$ \\
\hline Wavelength & 1.6513 & 1.6551 & 1.6597 \\
\hline $\mathrm{ZP} \mathrm{F}_{\lambda}$ at fiducial $\lambda\left(\mathrm{W} \mathrm{cm}^{-2} \mu \mathrm{m}^{-1}\right)$ & $1.182 \times 10^{-13}$ & $1.174 \times 10^{-13}$ & $1.163 \times 10^{-13}$ \\
\hline $\mathrm{ZP} \mathrm{F}_{v}$ at fiducial $\lambda(\mathrm{Jy})$ & 1075 & 1073 & 1068 \\
\hline Color correction/9550 K black body & 1.014 & 1.023 & \\
\hline Band & $K_{S}$ & & \\
\hline Band width & $0.2620 \mu \mathrm{m}$ & & \\
\hline Average $\mathrm{ZP}\left(\mathrm{W} \mathrm{cm}^{-2} \mu \mathrm{m}^{-1}\right)$ & $4.37 \times 10^{-14}$ & & \\
\hline Wavelength type & $\lambda_{0}(\mu \mathrm{m})$ & $\lambda_{0}^{\prime}(\mu \mathrm{m})$ & $\lambda_{\text {iso }}(\mu \mathrm{m})$ \\
\hline Wavelength & 2.1657 & 2.1692 & 2.1598 \\
\hline $\mathrm{ZP} \mathrm{F} \lambda$ at fiducial $\lambda\left(\mathrm{W} \mathrm{cm}^{-2} \mu \mathrm{m}^{-1}\right)$ & $4.32 \times 10^{-14}$ & $4.30 \times 10^{-14}$ & $4.36 \times 10^{-14}$ \\
\hline $\mathrm{ZP} \mathrm{F}_{v}$ at fiducial $\lambda(\mathrm{Jy})$ & 676 & 675 & 678 \\
\hline Color correction/9550 K black body & 1.018 & 1.030 & \\
\hline
\end{tabular}

very consistent behavior of solar-type and A-type stars, and that they in turn are closely consistent with virtually all direct calibration measurements and with models of their spectra. Concerns of significant inconsistencies (Price 2004; Bohlin 2007) can therefore be put aside, and we can proceed to develop a procedure for calibration of IR measurements with assurance that there are unlikely to be serious undetected systematic errors.

We have therefore established a consistent calibration across the near- and mid-IR spectral regions (1 to $25 \mu \mathrm{m})$. The foundation of the calibration is the accurate direct measurements near $2.2 \mu \mathrm{m}$ and particularly near $10 \mu \mathrm{m}$. The accuracy of the absolute calibration is $2 \%$ or better across this entire wavelength range. We provide guidelines for applying it to 2MASS, IRAC, and MIPS photometry. Because of the overall agreement among the previous calibrations, the adjustments to apply to them for a fully consistent IR calibration are small, generally within the stated errors.

After the rejection of a few stars with anomalous SEDs, upper limits of $\sim 1.5 \%$ (rms) are placed on the intrinsic IR SED variations in both A dwarf and solar-type stars. These types of star are therefore suitable as general-purpose standard stars, allowing the calibration to be extended readily to other photometric bands and systems. We provide SEDs of a fiducial A star and of the Sun for use in extending the calibration to other systems, or for generating fainter or brighter mid-IR standards by extrapolation from accurate near-IR measurements.

The suggested calibration is summarized in a number of tables. Table 1 gives the ZPs at 10.6 and $23.675 \mu \mathrm{m}$. Tables 9 and 10 provide a list of accurate measurements of $A$ and solar-type stars at the latter wavelength. Table 8 provides measurements of solar-type stars at $7.872 \mu \mathrm{m}$. These measurements can be transferred to any wavelength using the SEDs in Table 7. The listed stars are bright enough that they can be measured at high signal-to-noise from the ground at least through the $10 \mu \mathrm{m}$ atmospheric window, so they provide a direct transfer between
Table 7

Reference SEDs of the Sun and "Vega"

\begin{tabular}{lcc}
\hline $\begin{array}{l}\text { Wavelength } \\
(\mu \mathrm{m})\end{array}$ & $\begin{array}{c}\text { Sun } \\
\left(\mathrm{W} \mathrm{m}^{-2} \mathrm{~nm}^{-1}\right)\end{array}$ & $\begin{array}{c}\text { "Vega" } \\
\left(\mathrm{W} \mathrm{m}^{-2} \mathrm{~nm}^{-1}\right)\end{array}$ \\
\hline 0.1998 & $7.520 \times 10^{-3}$ & $5.581 \times 10^{-11}$ \\
0.2017 & $8.160 \times 10^{-3}$ & $5.312 \times 10^{-11}$ \\
0.2035 & $9.120 \times 10^{-3}$ & $5.165 \times 10^{-11}$ \\
0.2052 & $1.077 \times 10^{-2}$ & $5.752 \times 10^{-11}$ \\
\hline
\end{tabular}

(This table is available in its entirety in a machine-readable form in the online journal. A portion is shown here for guidance regarding its form and content)

the Spitzer calibration and ground-based observations. ZPs for 2MASS photometry are provided in Table 6.

In many cases, however, it is more convenient simply to adjust measurements using alternative calibrations to the consistent scale suggested in this paper. The relevant correction factors for 2MASS $K_{S}$, IRAC Band 4 and IRAS Bands 1 and 2 are listed in Table 5. Corrections to the other 2MASS and IRAC bands should be similar to those listed.

Previous work has suggested an inconsistency between IR and visible measurements of Vega, when fitted to a standard A0V star model. The improved accuracy of the IR calibration, and its confirmation through many approaches, makes it clear that this inconsistency is real. The A0V star model normalized to the IR calibration and extended to the visible $(0.5556 \mu \mathrm{m})$ predicts a flux density $4.2 \%=0.045$ mag brighter than the absolute measurements of Vega near this wavelength. We have independently verified this result by transforming the Vega measurements of Johnson et al. (1966) into the 2MASS system, showing that Vega would be $\sim-0.036$ at 2 MASS $K_{S}$. It is likely that the discrepancy has roots in Vega being a rapidly rotating star seen pole-on, so that its output spectrum is affected by the surface temperature gradient associated with the rapid 
rotation (Gulliver et al. 1994). We conclude that the $V-K_{S}$ color of Vega is about +0.045 mag (plus 0.014 mag to account for the excess due to its ring at $2 \mu \mathrm{m}$ ) compared with the average colors of A0V stars, and that this star is not suitable to define a photometric ZP because of its eccentric SED for its spectral type.

An important feature of our approach is its use of large, homogeneous databases-for example, Hipparcos and 2MASS photometry, the Nstars classification of nearby-solar-type stars, and the FEPS and MIPS GTO samples of solar-type and A-type stars observed to identify debris disks. The homogeneity and generally high accuracy of these data support a new approach to calibration. Initially large samples of stars are cleaned of members with anomalous colors (due, e.g., to reddening or photometric errors) and the results of still moderately large samples are then averaged to drive the measurement errors to small values. In addition to providing an accurate calibration that does not depend on a small number of "ideal" objects such as Vega was once thought to be, this approach facilitates extending the calibration over the entire sky.

We thank Tom Ayres, Martin Cohen, Chris Corbally, Mark Kidger, Gerry Neugebauer, Stephen Price, Murray Silverstone, and Gerard Thuillier for helpful discussions. This publication makes use of data products from the Two Micron All Sky Survey, which is a joint project of the University of Massachusetts and the IR Processing and Analysis Center/California Institute of Technology, funded by the National Aeronautics and Space Administration and the National Science Foundation. This research also has made use of the SIMBAD database, operated at CDS, Strasbourg, France. It also made use of the NASA/IPAC IR Science Archive. This work was supported through contracts 1255094 issued by JPL through CalTech and NAG5-12318 from NASA/Goddard to the University of Arizona.

\section{APPENDIX A}

This appendix provides the reference SEDs for the Sun and "Vega," from 0.2 to $30 \mu \mathrm{m}$. A sample of the first few entries is given in Table 7.

\section{APPENDIX B}

\section{LINEARITY}

The linearity of the IRAC measurements is discussed by Reach et al. (2005) and is adequate for the calibration we have derived. Here, we use the understanding gained regarding stellar colors to test whether there are any nonlinearities at a level that would affect the MIPS measurements. Because we have concentrated on stars measured at very high signal-to-noise at $24 \mu \mathrm{m}$ and also measured in the "Read 1" mode with 2MASS, the dynamic range of the measurements used in the calibrations in Sections 2 and 3 is small, only about a factor of 5, and hence the demands on instrument linearity are modest.

To test the linearity over a larger dynamic range, we use A stars from $\mathrm{Su}$ et al. (2006) that pass all the tests for our calibration sample, except that they are too bright to be measured by 2MASS in the Read 1 mode. Modern array detectors generally saturate on such bright stars (not just for 2MASS), so we depend on aperture photometry for the $K$-band data. Using these measurements requires that the photometric system be understood well enough to transfer accurately to the 2MASS Read 1 system. We have taken measurements of HD 18978, HD 108767, HD 130841, HD 135742, and HD 209952 from Carter (1990), of HD 80007 and HD 130841 from Bouchet et al. (1991), and of HD 11636, HD 16970, HD 76644, HD 87901, HD 103287, and HD 108767 from Johnson et al. (1966). Transformations for the first two references were obtained from Carpenter (2001). For the third reference, we first determined a transformation into the CIT system of $K_{\mathrm{AZ}}-K_{\mathrm{CIT}}=0.024$ from the table of bright-star measurements in Elias et al. (1982), and then independently confirmed the transformation in Carpenter (2001) from the table of fainter standards. Thus, the net transformation to 2MASS Read 1 observations is $K_{A Z}-K_{2 \mathrm{MASSR} 1}=0.048$, very close to the value of 0.044 derived independently by Bessell et al. (1998). The agreement of our Read-1 transformation with that of Carpenter (2001) validates our using his values for the other systems.

Figure 3 shows the normalized ratio of $24 \mu \mathrm{m}$ to $\mathrm{K}$ flux density, versus $24 \mu \mathrm{m}$ flux density. We exclude the two lowest measurements (of HD 172728). We also exclude the measurements of HD 47332 and HD 57336, the two highest measurements, because they may have faint excess emission. Since we have excluded the two highest and two lowest measurements, the bias on the results should be minimal.

We have considered three types of nonlinearity. The first is the typical gradual reduction of response in a simple integrating amplifier as the wells fill. The second is an over-correction for a nonlinearity of the first kind, so it just reverses the sign of the curve. The third is having a small latent image under the image of the star being measured. Since we have used custom flat fields to remove latents, the sign of the latent image could be either positive (adding to the star signal) or negative (subtracting from it). Examination of our data indicates that we can place an upper limit of about $50 \mu \mathrm{Jy}$ on any residual latent image.

As shown in Figure 3, the primary evidence for nonlinearity is a tendency for the bright stars to yield slightly larger signals than would be the case with a perfectly linear system. This offset is within the errors, but, if confirmed, it suggests that the correction for nonlinearity in large signals may be slightly too big (see Engelbracht et al. 2007 for further discussion). There is also evidence for a slight tendency to obtain larger net slopes with increasing integration time, an effect that would be consistent with the possible nonlinearity (Engelbracht et al. 2007). However, at the level of our measurements, 10-50 mJy, these effects are negligible.

\section{APPENDIX C}

\section{MEASUREMENTS WITH SPITZER}

This appendix discusses our measurement procedures for the Spitzer data. It also collects the samples of stars used for the various calculations in the paper, along with their key parameters.

\section{C.1. Procedures at $8 \mu \mathrm{m}$}

One of us (J. Carpenter) re-reduced the measurements of our solar-type stars to put them on the identical basis as the calibration reductions of Reach et al. (2005). The $8 \mu \mathrm{m}$ solar analog calibration is based on the stars listed in Table 8 . Fortunately, although some of the IRAC bands can have small offsets due to positioning of the measuring aperture, this effect is immeasurably small at $8 \mu \mathrm{m}$ (M. Silverstone 2006, private communication), and we have ignored it. We thus only had to divide by the bandpass correction to convert to equivalent 


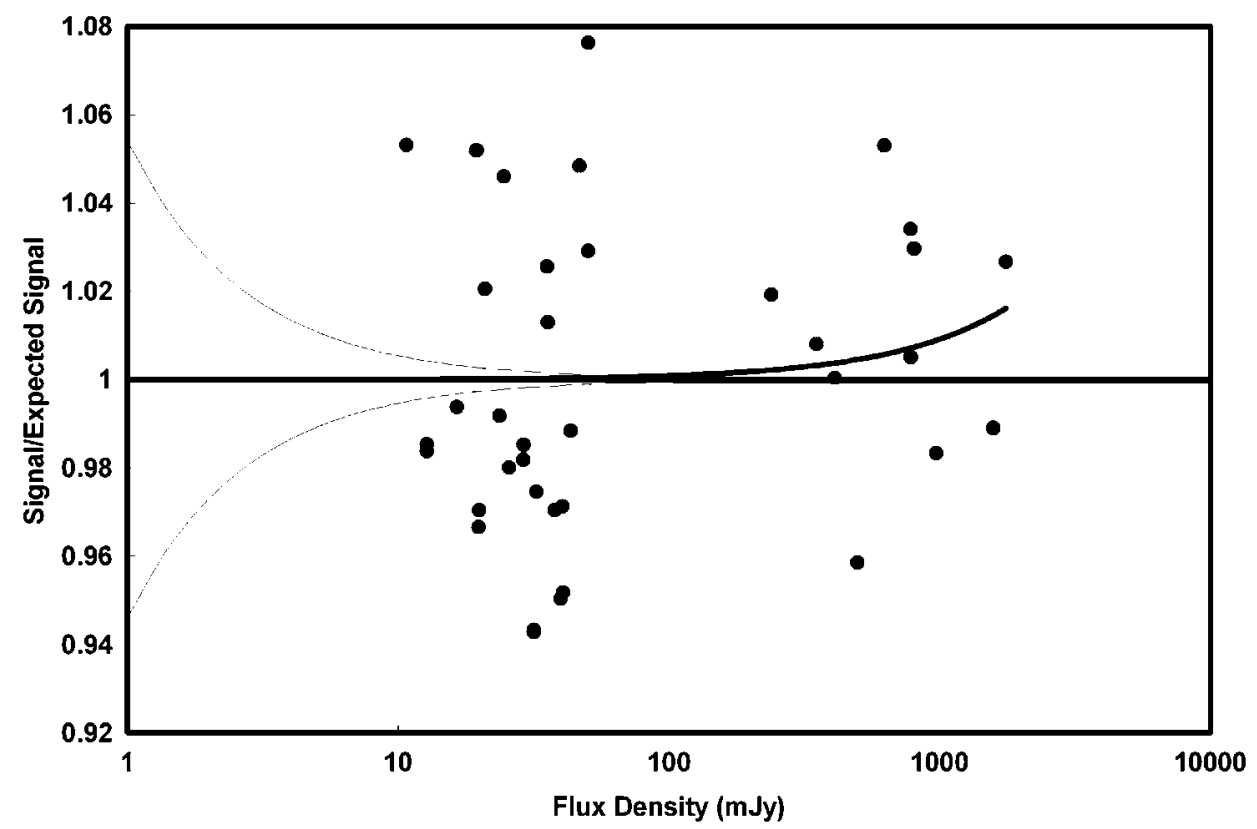

Figure 3. Normalized (to linear response) signal versus flux density at $24 \mu \mathrm{m}$. Models for three different types of nonlinearity are compared with the data. The two light lines are nonlinearity due to residual positive or negative $50 \mu \mathrm{Jy}$ latent images. The heavy line is a linearity correction; the best fit suggests that the correction applied in the data pipeline may be slightly too large (but not at a significant level). However, there is no convincing argument for any significant nonlinearity over the range of the calibration measurements $(10-50 \mathrm{mJy})$.

monochromatic flux density at $7.872 \mu \mathrm{m}$. Since the SEDs of solar-type stars match very closely in this spectral region those of A stars, we applied the correction quoted by Reach et al. for an A1 star, 1.042 .

\section{C.2. Procedures at $24 \mu \mathrm{m}$}

To achieve the most accurate possible data reduction at $24 \mu \mathrm{m}$, we have used a series of custom processing steps that have been developed by the MIPS team (discussed in more detail by Engelbracht et al. 2007). These steps are applied after standard pipeline processing. First, we remove the artifacts due to dust particles on the instrument pick-off mirror. Special flat fields are constructed from all of the photometric data to identify the effects of these particles. Second, we compensate for the latent images on the $24 \mu \mathrm{m}$ array as a result of exposure to bright sources. These dark latents appear as regions of reduced sensitivity centered on the array positions of bright sources in subsequent exposures. An image of the latents with all sources removed is produced by median combining the entire data set with appropriate bright source masks. The dark latents are removed from each individual frame by dividing the frames by the normalized dark latent image prior to mosaicking. ${ }^{12}$ Finally, mosaicked images are produced at the nominal pixel scale, $2.45^{\prime \prime}$. This processing is described in more detail by Engelbracht et al. (2007).

We used simple aperture photometry on the $24 \mu \mathrm{m}$ images. The tabulated data assumed $1 \mathrm{DN} \mathrm{s}^{-1}=1.05 \mathrm{mJy} \operatorname{arcsec}^{-2}$. The photometry was done within an aperture of radius $35^{\prime \prime}$, and relative to sky measured in an annulus between radii of 40 and $50^{\prime \prime}$. The aperture correction for this measurement approach was taken to be 1.084 . We used the standard world coordinate

12 A mean background frame determined from the masked data is subtracted as well. The mean of the subtracted values is added back to each frame. This step removes a small gradient likely due to scattered zodiacal light that depends on the position of the scan mirror. system pointing information as a first guess for centering, with fine tuning with the IDL program "mpfit2dpeak.pro" written by Craig Markwardt. Comparing photometry for separate measurements of the same star (typically also in different observation campaigns) indicates that the end-to-end scatter (including instrumental instability as well as photometry errors) is less than $0.5 \% \mathrm{rms}$ (and not dependent on the brightness of the target for the range considered here-further discussion is in Engelbracht et al. 2007). Achieving this level of repeatability requires not only the special post-pipeline processing, but also careful standardization of photometric procedures. Therefore, all the results reported in this paper used the identical photometric procedures applied by the same person (M. Blaylock). The results are listed in Tables 9 and 10; the maximum plausible errors in this band are no more than $1.5 \%$ rms.

Our procedures have been selected to be very conservative (e.g., large measurement aperture) and adapted to high repeatability. Other photometry approaches can be tested by using archival data for our program stars listed in Tables 9 and 10.

\section{C.3. Color Combinations}

Tables 8-10 list the 2MASS $K_{S}$ value and a second $K_{S}$ magnitude. Super $K_{S}$ is a combination of the 2MASS $J, H$, and $K_{S}$ measurements used to measure the scatter in $K_{S}-$ [24] color among the stars. We used the standard colors for the spectral type of the star (Tokunaga 2000) to convert the $J$ and $H$ measurements to equivalent $K_{S}$ ones. The typical distance to one of the A stars is about $100 \mathrm{pc}$, so reddening may be significant. We estimated the reddening from the standard $V-K$ color for the spectral type of the star (Tokunaga 2000) and the extinction curve of Rieke \& Lebofsky (1985), and corrected all the $J, H$, and $K$ measurements accordingly (the $24 \mu \mathrm{m}$ extinction is less than $1 \%$ even for the most obscured of the stars). The Super $K_{S}$ magnitude is the weighted average of all three extinction- and color-corrected measurements. Because the colors of A stars 
Table 8

Solar-Type $8 \mu \mathrm{m}$ Calibration Sample

\begin{tabular}{lccccc}
\hline \hline Name & Type & $\begin{array}{c}\text { Super } K_{S} \\
(\mathrm{mag})\end{array}$ & $\begin{array}{c}K_{S} \\
(\mathrm{mag})\end{array}$ & $\begin{array}{c}f(8 \mu \mathrm{m}) \\
(\mathrm{mJy})\end{array}$ & $\begin{array}{c}\text { Flux ratio }^{\mathrm{a}} \\
\end{array}$ \\
& & & & & \\
\hline HD 00643 & G2/G3V & 6.204 & 6.187 & 215.1 & 0.99 \\
HD 00894 & F8IV-V & 5.353 & 5.378 & 448.8 & 0.98 \\
HD 01901 & F8 & 5.563 & 5.57 & 371.6 & 0.97 \\
HD 02746 & G5 & 6.262 & 6.282 & 198.5 & 1.00 \\
HD 03796 & G4V & 6.279 & 6.268 & 198.2 & 0.98 \\
HD 03894 & G1V & 6.419 & 6.442 & 172.3 & 1.00 \\
HD 06073 & G0 & 6.246 & 6.253 & 202.1 & 0.99 \\
HD 08820 & G0IV-V & 6.114 & 6.118 & 229.9 & 0.99 \\
HD 08874 & G1V & 4.967 & 4.955 & 663.2 & 0.98 \\
HD 09071 & G2/G3V & 6.054 & 6.042 & 235.4 & 0.95 \\
HD 09278 & G5 & 5.724 & 5.721 & 322.7 & 0.96 \\
HD 09855 & G2/G3V & 6.072 & 6.078 & 238.8 & 0.99 \\
HD 10195 & G0V & 5.611 & 5.609 & 361.3 & 0.97 \\
HD 10625 & G0V & 5.940 & 5.929 & 270.9 & 0.98 \\
HD 10879 & G1/G2V & 4.857 & 4.832 & 767.1 & 1.01 \\
HD 10894 & F8 & 6.009 & 6.025 & 256.9 & 1.02 \\
HD 11219 & F8V & 5.556 & 5.553 & 395.6 & 1.01 \\
HD 11504 & G1Va & 5.331 & 5.334 & 476.0 & 1.00 \\
HD 12150 & G2V & 6.135 & 6.124 & 225.3 & 0.98 \\
HD 12265 & F8 & 5.863 & 5.879 & 283.1 & 0.98 \\
HD 14193 & G2/G3V & 5.771 & 5.764 & 310.8 & 0.97 \\
HD 15070 & G0 & 5.572 & 5.565 & 388.1 & 1.01 \\
HD 15922 & G5V & 4.981 & 4.998 & 613.3 & 0.94 \\
HD 17994 & F8V & 4.952 & 4.936 & 658.4 & 0.96 \\
HD 18321 & G2V & 5.754 & 5.766 & 320.8 & 1.00 \\
HD 19301 & F8 & 5.945 & 5.966 & 264.7 & 0.99 \\
HD 19503 & G5 & 5.561 & 5.579 & 379.4 & 1.00 \\
HD 19959 & G0V & 5.462 & 5.461 & 412.2 & 0.97 \\
HD 20427 & F8V & 5.412 & 5.449 & 415.8 & 0.97 \\
HD 20590 & G4IV-V & 5.314 & 5.321 & 491.1 & 1.02 \\
HD 21229 & G5 & 6.265 & 6.269 & 206.0 & 1.02 \\
HD 21627 & G0 & 5.782 & 5.794 & 309.5 & 0.99 \\
\hline & & & & & \\
\hline
\end{tabular}

Notes.

${ }^{a}$ Ratio of observed flux density to expected flux density from the photosphere. See Rieke et al. (2005) and Su et al. (2006) for further details.

are close to zero already, minor differences in the photometric system have little influence on this conversion. The small offsets in the 2MASS $J-K$ and $H-K$ colors discussed in Appendix D have a negligible effect on our calculations here, because they are nearly equal and opposite in sign. The nominal errors in the resulting Super $K_{S}$ magnitudes are $2 \%$ or less. Similar procedures were used to compute Super $K_{S}$ for the solar-type stars in Tables 8 and 10, except that no extinction corrections were applied. Although some of the minor offsets in 2MASS have been mitigated by our using only Read 1 observations, there is little information over the full Read 1 dynamic range on how the photometry behaves at this level of accuracy. We therefore place an estimate of $3 \%$ on the net errors-half from the rms errors and half from possible systematic ones.

\section{C.4. Procedure with IRS Data}

To obtain a high signal-to-noise IRS spectrum of a solar-type star, we started with the spectra of the A stars HR 1014, 2194, 5467, and HD 163466 and of the solar-type stars HD 9826, 10800, 13974, 39091, 55575, 84737, 86728, 95128, 133002, $136064,142373,188376,196378,212330$, and 217014 obtained from the Spitzer archive. After standard processing with the S13
Table 9

A-Type Stars Used to Determine the $K_{S}-[24] \mathrm{ZP}$

\begin{tabular}{|c|c|c|c|c|c|c|}
\hline Name & $\begin{array}{c}\text { Age } \\
(\mathrm{Myr})\end{array}$ & $\begin{array}{c}V \\
\text { (mag) }\end{array}$ & $\begin{array}{c}\text { Super }_{S} \\
\text { (mag) }\end{array}$ & $\begin{array}{c}K_{S} \\
(\mathrm{mag})\end{array}$ & $\begin{array}{c}f(24 \mu \mathrm{m}) \\
(\mathrm{mJy})\end{array}$ & Flux ratio \\
\hline HD 319 & 600 & A1V & 5.467 & 5.479 & 45.89 & 0.99 \\
\hline HD 02811 & 750 & A3V & 7.067 & 7.057 & 10.94 & 1.01 \\
\hline HD 11413 & 600 & A1V & 5.396 & 5.422 & 53.13 & $1.08^{\mathrm{b}}$ \\
\hline HD 14943 & 850 & A5V & 5.469 & 5.439 & 48.92 & 1.01 \\
\hline HD 15646 & 260 & A0V & 6.398 & 6.411 & 20.15 & 1.02 \\
\hline HD 17254 & 650 & $\mathrm{~A} 2 \mathrm{~V}$ & 5.907 & 5.877 & 31.12 & 0.97 \\
\hline HD 17254 & 650 & $\mathrm{~A} 2 \mathrm{~V}$ & 5.907 & 5.877 & 30.47 & 0.94 \\
\hline HD 20888 & 300 & $\mathrm{~A} 3 \mathrm{~V}$ & 5.723 & 5.691 & 37.02 & 0.97 \\
\hline HD 20888 & 300 & A3V & 5.744 & 5.691 & 38.28 & 1.00 \\
\hline HD 21981 & 265 & A1V & 5.513 & 5.526 & 41.35 & 0.93 \\
\hline HD 34868 & 300 & $\mathrm{~A} 0 \mathrm{~V}$ & 6.018 & 6.024 & 26.3 & 0.93 \\
\hline HD 42525 & 300 & $\mathrm{~A} 0 \mathrm{~V}$ & 5.763 & 5.751 & 34.76 & 0.96 \\
\hline HD 57336 & 400 & A0IV & 7.188 & 7.114 & 9.544 & 0.92 \\
\hline HD 73210 & 729 & A5V & 6.170 & 6.165 & 24.96 & 1.01 \\
\hline HD 73666 & 729 & A1V & 6.524 & 6.532 & 18.11 & 1.03 \\
\hline HD 73819 & 729 & A6Vn & 6.322 & 6.28 & 21.42 & 0.96 \\
\hline HD 92845 & 300 & $\mathrm{~A} 0 \mathrm{~V}$ & 5.519 & 5.513 & 47.71 & $1.06^{\mathrm{b}}$ \\
\hline HD 101452 & 250 & $\mathrm{~A} 2$ & 6.799 & 6.819 & 13.01 & 0.96 \\
\hline HD 105805 & 500 & $\mathrm{~A} 4 \mathrm{Vn}$ & 5.626 & 5.6 & 38.96 & 0.94 \\
\hline HD 116706 & 500 & A3IV & 5.507 & 5.502 & 42.03 & 0.92 \\
\hline HD 128998 & 250 & A1V & 5.758 & 5.756 & 32.73 & 0.91 \\
\hline HD 158485 & 420 & A $4 \mathrm{~V}$ & 6.153 & 6.145 & 24.30 & 0.96 \\
\hline HD 163466 & 310 & A2 & 6.300 & 6.339 & 19.48 & 0.92 \\
\hline HD 163466 & 310 & $\mathrm{~A} 2$ & 6.300 & 6.339 & 20.03 & 0.95 \\
\hline HD 172728 & 210 & $\mathrm{~A} 0 \mathrm{~V}$ & 5.746 & 5.753 & 32.33 & $0.89^{c}$ \\
\hline HD 172728 & 210 & $\mathrm{~A} 0 \mathrm{~V}$ & 5.746 & 5.753 & 32.3 & $0.89^{c}$ \\
\hline
\end{tabular}

Notes.

a Ratio of observed flux density to expected flux density from the photosphere. See Rieke et al. (2005) and Su et al. (2006) for further details.

b Possible weak $24 \mu \mathrm{m}$ excess.

c Low value probably reflects a measurement error, possibly in the 2MASS $K_{S}$ magnitude.

IRS pipeline to the $\mathrm{BCD}$ level, nod pairs were subtracted to remove the background and the spectra were extracted with SPICE (Spitzer Science Center 2007). We then ratioed the spectra for similar stellar types in various combinations to determine the subset that were most closely similar. We also gave weight to evidence from the signal strength that the star was accurately centered in the spectrograph slit. As a result, we rejected HR 1014 from further processing because of a number of broad peaks and valleys in the ratio. We rejected the short module data for HD 9826, 95128, 188376 because of pronounced downward slopes with increasing wavelength in the ratios, and also HD 13974 and HD 196378 because the S module ratio is low relative to the $\mathrm{L}$ module data, suggesting a pointing issue with S. We rejected the L module data for HD 13974 and 188376 because of slopes and curves in the ratios, as well as for HD 9826, 95128, and HD 142373 because low values of the ratio relative to that for the $\mathrm{S}$ module suggest pointing issues. We averaged the accepted spectra (ten for each module) with equal weight, and then divided the solar-type average spectrum with the A-star one and multiplied the result by the A-star Kurucz model. The resulting spectrum had a discontinuity between the short and long IRS modules, which we removed by forcing the average value between 12 and $14 \mu \mathrm{m}$ to equal that between 15 and $17 \mu \mathrm{m}$. We finally smoothed the result with a 7 pixel boxcar (giving a final spectral resolution of $\sim 10 \%$ ). 
Table 10

Solar-Type $24 \mu \mathrm{m}$ Calibration Sample

\begin{tabular}{|c|c|c|c|c|c|c|}
\hline Name & $\begin{array}{c}\text { Age } \\
(\mathrm{Myr})\end{array}$ & Type & $\begin{array}{c}\text { Super }_{S} \\
(\mathrm{mag})\end{array}$ & $\begin{array}{c}K_{S} \\
(\mathrm{mag})\end{array}$ & $\begin{array}{c}f(24 \mu \mathrm{m}) \\
(\mathrm{mJy})\end{array}$ & Flux ratio $^{a}$ \\
\hline HD 008941 & 2200 & F8IV-V & 5.353 & 5.378 & 49.2 & 0.96 \\
\hline HD 019019 & 1700 & F8 & 5.563 & 5.57 & 42.4 & 0.99 \\
\hline HD 027466 & 1500 & G5 & 6.262 & 6.282 & 21.5 & 0.97 \\
\hline HD 037962 & 2400 & G4V & 6.279 & 6.268 & 22.4 & 1.00 \\
\hline HD 038949 & 1000 & G1V & 6.419 & 6.442 & 19.6 & 1.02 \\
\hline HD 064324 & 1700 & G0 & 6.234 & 6.235 & 25.1 & 1.08 \\
\hline HD 066751 & 4200 & F8 & 5.061 & 5.066 & 66.1 & 0.97 \\
\hline HD 092788 & 6800 & G5 & 5.724 & 5.721 & 36.2 & 0.97 \\
\hline HD 098553 & 5800 & G2/G3V & 6.072 & 6.078 & 26.4 & 0.98 \\
\hline HD 100167 & 2300 & F8 & 5.826 & 5.806 & 34.4 & 1.00 \\
\hline HD 101472 & 1100 & G0 & 6.125 & 6.139 & 26.2 & 1.03 \\
\hline HD 101959 & 4700 & G0V & 5.611 & 5.609 & 39.8 & 0.96 \\
\hline HD 106252 & 5000 & G0V & 5.940 & 5.929 & 32.4 & 1.06 \\
\hline HD 108799 & 9300 & G1/G2V & 4.857 & 4.832 & 84.9 & 1.01 \\
\hline HD 108944 & 4500 & F8 & 6.009 & 6.025 & 30.3 & 1.08 \\
\hline HD 112196 & 7300 & F8V & 5.556 & 5.553 & 43.4 & 1.00 \\
\hline HD 115043 & 11500 & G1Va & 5.331 & 5.334 & 53.7 & 1.01 \\
\hline HD 121504 & 7100 & $\mathrm{G} 2 \mathrm{~V}$ & 6.135 & 6.124 & 27.4 & 1.07 \\
\hline HD 122652 & 2500 & F8 & 5.863 & 5.879 & 34.8 & 1.08 \\
\hline HD 141937 & 4500 & G2/G3V & 5.771 & 5.764 & 35.0 & 0.98 \\
\hline HD 150706 & 1400 & G0 & 5.572 & 5.565 & 45.5 & 1.06 \\
\hline HD 153458 & 1400 & G0 & 6.449 & 6.447 & 19.1 & 1.00 \\
\hline HD 159222 & 3800 & G5V & 4.981 & 4.998 & 68.3 & 0.94 \\
\hline HD 167389 & 2200 & F8 & 5.910 & 5.918 & 30.4 & 0.98 \\
\hline HD 193017 & 1900 & F8 & 5.945 & 5.966 & 28.4 & 0.95 \\
\hline HD 195034 & 4800 & G5 & 5.561 & 5.579 & 41.9 & 0.99 \\
\hline HD 199598 & 2100 & G0V & 5.462 & 5.461 & 48.0 & 1.01 \\
\hline HD 204277 & 1100 & F8V & 5.412 & 5.449 & 49.9 & 1.04 \\
\hline HD 205905 & 1500 & G4IV-V & 5.314 & 5.321 & 53.8 & 1.00 \\
\hline HD 212291 & 2900 & G5 & 6.265 & 6.269 & 22.7 & 1.01 \\
\hline HD 216275 & 4400 & G0 & 5.782 & 5.794 & 34.6 & 0.99 \\
\hline
\end{tabular}

Notes.

a Ratio of observed flux density to expected flux density from the photosphere. See Rieke et al. (2005) and Su et al. (2006) for further details.

\section{APPENDIX D}

\section{$J H K$ SYSTEM}

\section{D.1. Vega and the Zero Point}

Most near-IR photometric systems claim to be relative to a ZP defined by Vega, with this star or a model of it placed either at 0.02 (to coincide with the Johnson bright-star measurements) or at zero. We have investigated how the peculiarities of this star have affected these definitions. This question is not easily answered because few of the sets of photometry since Johnson have actually measured Vega directly. We have therefore evaluated the quality of the Johnson photometry and then transformed it into the 2MASS system, finally using the transformation to compute the magnitude of Vega as it would have been measured by 2 MASS.

Johnson et al. (1966) report 173 measurements of Vega within the overall set of photometry that constitutes their study of bright stars. We evaluated the quality of this photometry in two ways. First, we looked at the internal scatter for the individual measurements of Vega. We rejected the four highest and lowest measurements at both $J$ and $K$ (i.e., $4.6 \%$ of the measurements) and computed the straight average and standard deviation of the remaining 165 measurements. The results are $J=0.019 \pm 0.004, K=0.023 \pm 0.004$, and rms scatter of 0.048 and 0.050 in $J$ and $K$, respectively. With no outlier rejection the values for Vega are unaffected, but the errors increase to 0.005 and the rms scatter values to 0.061 and 0.065 . However, we believe the values with the outlier rejection are the most representative.

We have tested this result in another way, by comparing stars measured in common by Johnson et al. (1966) and either Bouchet et al. (1991) or Kidger \& Martín-Luis (2003). We first transformed the Johnson photometry into the other system (as described below). We excluded stars used by Johnson et al. (1966) as standards (stars such as Vega that were measured many times) in case their use in fitting for the photometric corrections would bias the comparison. There were 314 measurements suitable for the comparison with Bouchet et al. (1991), of which we rejected the seven high and seven low outliers (i.e., $4.5 \%$ of the measurements). The rms of the deviations was then 0.054 at $J$ and 0.048 at $K$, that is, in excellent agreement with the internal scatter of the Johnson measurements of Vega. Similarly for the measurements of Kidger \& Martín-Luis (2003), there were 135 suitable measurements. After transforming the Johnson photometry and rejecting the two high and two low outliers (3\% of the measurements), the rms scatter is 0.048 at both $J$ and $K$. We conclude that the Johnson et al. (1966) photometry is valid at the level of $1-\sigma$ errors of 0.05 mag for single measurements. Since most of the published bright-star photometry is based on three or more measurements per star, this result is consistent with a typical overall accuracy of $3 \%$ or better.

The accuracy of the Johnson et al. (1966) photometry implies that Vega is tied into their overall photometric system to a $1-\sigma$ error of less than $0.005 \mathrm{mag}$. We will now determine transformations to put this measurement into the 2MASS Read 1 system. The simplest approach is to transfer directly. However, there are relatively few stars that were measured to high accuracy with the original Johnson photometer and that are faint enough not to saturate the 2MASS detector arrays. We have based the transformation on stars measured in Johnson et al. (1966, 1968) and in Lee (1968). The comparison is compromised by the decreased signal-to-noise of the stars and we have not attempted a color correction (although the data imply it would be small). We derive that Vega would have a 2MASS $J$ magnitude of -0.042 and a $K$ magnitude of -0.057 . Given the issues with signal-to-noise, we prefer the average of these values, -0.050 , as a best estimate of the near-IR Vega magnitude.

This result is limited by the poor overlap in the dynamic range of the two measurement sets; to overcome this problem, we have calculated the 2MASS Vega magnitudes by transforming the Johnson photometry to other systems that have a wider overlap with 2MASS. For the first case, we used bright standards in the CIT system defined by Elias et al. (1982). We find a transformation in good agreement with those derived previously by Elias et al. (1985) and Bessell \& Brett (1988), although our procedure differed from theirs because we fitted the individual measurements of Johnson et al. (1966) not the final photometric results combining those measurements for each star. We use the transformation from the CIT to 2MASS systems available at the 2MASS Web site to obtain 2MASS $K_{S}$ magnitudes for Vega of $-0.028,-0.023$, and -0.032 using respectively our transformation and those of Bessell \& Brett (1988) and of Elias et al. (1985). Similarly, we obtain a 2 MASS $K_{S}$ magnitude of -0.033 if we make the transformation through the ESO system photometry of Bouchet et al. (1991) and a $K_{S}$ magnitude of -0.045 if the transformation is made through the main body of photometry of Kidger \& Martín-Luis (2003; their Table 2). 
There is a conflict in the latter case because Kidger \& MartínLuis (2003) also measured Vega directly, using a reduced system gain, and set it identically to zero magnitude at the near-IR bands (their Table 3 ). To check this result, we have computed the average $K-L$ (rejecting one high and one low value) for the 19 late B stars and A stars using $L$ from their Table 3 (in all cases measured with the reduced gain) and $K$ from either Table 2 or 3 . We obtain $+0.058 \pm 0.016$ where we would have expected zero. This result implies that the $L$ magnitude of Vega if measured relative to the high-gain $K$ photometry would be $-0.058 \pm 0.016$, in agreement with the negative $K$ magnitude derived from their Table 2. Although we have been unable to explain the discrepancy completely, the behavior of the other stars at $L$ has led us to accept the value from the transformation of the data in Table 2. An additional comparison can be made from Koornneef (1983), who established a system based on the Johnson $J$ and $K$ photometry. From the 2MASS color transformations, the corresponding 2MASS $K$ magnitude for Vega would be -0.023 .

All of these estimates are consistent with assigning Vega the average $K$ magnitude of $-0.036 \pm 0.006$ in the 2 MASS system. The quoted error is based on the scatter of the measurements; given the small number of them, an error of 0.010 is a more secure estimate.

\section{D.2. 2MASS Measurements}

The 2MASS data provide a very homogeneous set of nearIR photometry over the entire sky, with accuracies well within the original specifications for the survey. Nonetheless, there are small offsets in those measurements that can be significant at the levels of accuracy desired for the calibration discussed in this paper. We discuss two such effects in this appendix.

\section{D.3. Read 1/Read 2 offset}

There are offsets of the order of $2 \%$ between Read 1 and Read 2 measurements, in the range where they overlap. It is not clear how to derive a universal correction for this effect. Therefore, for the calibration we selected stars measured only in the Read 1 mode.

\section{D.4. Procedures for VJHK Calibration of A Stars}

To define a ZP at $V, J, H$, and $K$, we built a sample of 57 A0V stars distributed over the entire sky, brighter than $m_{V}=$ 7 mag, and with "A" quality Read 12 MASS measurements at all three near-IR colors. We took $B$ and $V$ magnitudes from the Hipparcos Main Catalog (Perryman et al. 1997). Stellar classifications were taken from SIMBAD. The IR colors are not a strong function of the spectral type-from B8 to A2, $J-K$ changes from -0.03 to +0.03 (Tokunaga 2000)—so type errors are not a major concern. However, reddening is. To eliminate reddened stars (and other sources of error), we excluded those with $B-V>0.05$ and those with $\left|V-K_{S}\right|>0.05$. In addition, because the 2MASS quoted errors can be large in the $H$ band, we excluded three stars with $H-K>0.07$, which is $5 \%$ redder than the value averaged over the sample. For the final sample of 57 stars (see Table 11), the result was an average $\langle V-K\rangle$ consistent with zero, $\langle J-K\rangle=-0.022 \pm 0.003$, and $\langle H-K\rangle=0.019 \pm 0.003$. The average values are not affected by various assumptions we tried with regard to final sample selection. For example, if we keep all stars with $|V-K|<0.1$, we get a slight positive residual in $\langle V-K\rangle$, but
Table 11

Near-IR Colors of A Stars in 2MASS Read 1 Mode

\begin{tabular}{|c|c|c|c|c|c|}
\hline Name & $V$ & $B-V$ & $V-K$ & $J-K$ & $H-K$ \\
\hline HD 012468 & 6.52 & 0.003 & -0.037 & -0.05 & 0.016 \\
\hline HD 021379 & 6.29 & -0.025 & -0.021 & -0.029 & 0.01 \\
\hline HD 022789 & 6.01 & -0.027 & 0.002 & 0 & 0.057 \\
\hline HD 029526 & 5.66 & 0.002 & 0.011 & 0.014 & 0.019 \\
\hline HD 031069 & 6.06 & -0.032 & -0.047 & -0.059 & -0.004 \\
\hline HD 035505 & 5.64 & -0.001 & -0.009 & -0.002 & 0.029 \\
\hline HD 035656 & 6.41 & -0.023 & -0.028 & -0.044 & -0.02 \\
\hline HD 036473 & 5.53 & 0.012 & 0.016 & 0.032 & 0.006 \\
\hline HD 041076 & 6.09 & -0.035 & 0.002 & 0.004 & 0.035 \\
\hline HD 042301 & 5.49 & -0.01 & 0.037 & 0.008 & 0.034 \\
\hline HD 042729 & 6.08 & -0.021 & 0.051 & 0.004 & 0.05 \\
\hline HD 043583 & 6.59 & -0.036 & -0.031 & -0.057 & -0.009 \\
\hline HD 045137 & 6.51 & -0.027 & 0 & -0.011 & -0.005 \\
\hline HD 045557 & 5.78 & -0.001 & 0.026 & 0.001 & 0.049 \\
\hline HD 056386 & 6.19 & -0.024 & 0.018 & -0.042 & 0.016 \\
\hline HD 060629 & 6.64 & -0.007 & -0.015 & -0.03 & 0.027 \\
\hline HD 070175 & 7 & -0.01 & -0.009 & -0.001 & 0.035 \\
\hline HD 071043 & 5.89 & 0.018 & 0.017 & -0.011 & -0.002 \\
\hline HD 072337 & 5.51 & -0.024 & -0.04 & -0.008 & 0.028 \\
\hline HD 076346 & 6.02 & -0.023 & -0.024 & -0.004 & 0.003 \\
\hline HD 078955 & 6.53 & 0.001 & 0.041 & 0.018 & 0.062 \\
\hline HD 079108 & 6.14 & -0.01 & 0.021 & 0.023 & 0.044 \\
\hline HD 080950 & 5.86 & -0.024 & -0.005 & 0.035 & 0.055 \\
\hline HD 086087 & 5.71 & 0.006 & -0.027 & -0.035 & 0.028 \\
\hline HD 096338 & 6.82 & 0.043 & 0.051 & 0.009 & -0.007 \\
\hline HD 102981 & 6.62 & -0.027 & -0.021 & -0.026 & 0.03 \\
\hline HD 104430 & 6.16 & -0.004 & -0.034 & -0.01 & -0.015 \\
\hline HD 107655 & 6.21 & -0.012 & 0.043 & -0.049 & -0.029 \\
\hline HD 107947 & 6.61 & -0.001 & -0.008 & -0.037 & 0.032 \\
\hline HD 113457 & 6.64 & 0.006 & 0 & -0.032 & 0.007 \\
\hline HD 115527 & 6.87 & -0.006 & -0.007 & -0.032 & 0.011 \\
\hline HD 117651 & 6.36 & -0.014 & -0.006 & -0.044 & 0.03 \\
\hline HD 118214 & 5.6 & -0.014 & -0.035 & -0.035 & 0.018 \\
\hline HD 121409 & 5.7 & -0.032 & 0.022 & -0.03 & 0.03 \\
\hline HD 124683 & 5.53 & 0.001 & -0.022 & -0.009 & -0.004 \\
\hline HD 131951 & 5.9 & -0.031 & 0.001 & -0.048 & 0.02 \\
\hline HD 136831 & 6.29 & -0.008 & 0.042 & -0.031 & 0.008 \\
\hline HD 140729 & 6.15 & 0.002 & 0.019 & -0.054 & -0.016 \\
\hline HD 143488 & 6.99 & 0.006 & 0.023 & -0.022 & 0.042 \\
\hline HD 145122 & 6.13 & -0.003 & 0.046 & -0.032 & 0.005 \\
\hline HD 145454 & 5.44 & -0.019 & 0.009 & -0.058 & -0.003 \\
\hline HD 154972 & 6.24 & -0.004 & 0.02 & -0.011 & 0.014 \\
\hline HD 155379 & 6.52 & -0.037 & 0 & -0.007 & -0.029 \\
\hline HD 172728 & 5.74 & -0.045 & -0.013 & -0.028 & 0.02 \\
\hline HD 176425 & 6.21 & 0.001 & -0.018 & -0.024 & 0.064 \\
\hline HD 177406 & 5.95 & -0.017 & -0.014 & -0.006 & 0.031 \\
\hline HD 178207 & 5.4 & -0.014 & -0.014 & -0.046 & 0.032 \\
\hline HD 179933 & 6.26 & 0.008 & 0.001 & -0.048 & 0.003 \\
\hline HD 182761 & 6.31 & -0.014 & 0.023 & -0.045 & 0.031 \\
\hline HD 182919 & 5.6 & -0.006 & -0.013 & -0.038 & 0.014 \\
\hline HD 195549 & 6.35 & 0.001 & 0.007 & -0.007 & 0.017 \\
\hline HD 205314 & 5.77 & -0.042 & -0.018 & -0.055 & 0.025 \\
\hline HD 207636 & 6.45 & -0.007 & 0.033 & -0.015 & 0.026 \\
\hline HD 212643 & 6.29 & -0.021 & 0.005 & -0.028 & -0.006 \\
\hline HD 219290 & 6.32 & -0.005 & -0.001 & -0.024 & -0.006 \\
\hline HD 219485 & 5.89 & -0.01 & 0.018 & -0.035 & 0.026 \\
\hline HD 223386 & 6.33 & -0.007 & -0.001 & -0.03 & -0.005 \\
\hline
\end{tabular}

values for $\langle J-K\rangle$ and $\langle H-K\rangle$ equal to those above within the errors.

An independent test was conducted with A0 dwarfs from the Michigan spectral atlas. There are a total of 6008 such stars with "good" 2MASS measurements in all three bands. We discarded all of them with $\left|V-K_{S}\right|>0.05$, leading to a sample of 510 . 
Eleven of this sample were discarded as photometric outliers. Of the remaining stars, 220 have Read 1 photometry in all three bands, and the net $J-K_{S}=-0.016 \pm 0.018$ for these stars. Similarly, we found a net $H-K_{S}=+0.026 \pm 0.022$ based on 212 stars from the Michigan spectral atlas sample. The quoted errors are the population standard deviations. The nominal standard deviations of the means are only about 0.001 , but there are suggestions of other low-level trends in the data that would make such an accurate color difficult to obtain. We conclude that the net colors are in agreement with those derived from the smaller and more heterogeneous sample discussed above. The result for the stars measured in the Read 2 mode are $J-K_{S}=-0.005 \pm 0.021$ (population sigma, 150 stars) showing that the offset is a function of the 2MASS read mode. Therefore, corrections to remove these small effects will be relatively complex to apply. Cohen et al. (2003) have also identified offsets of similar size, but not with the same values as those we have determined. Their sample is predominantly $K$ giants, and their Vega ZP model is redder than ours. If we compute the offsets only for the nine A stars in their sample and correct for the different colors for Vega, their results agree with ours to within $1.5-2 \%$ with errors of about $1.6 \%$, i.e., within the errors.

These results could at least partially explain IR color offsets such as those found by Casagrande et al. (2006; their Figures 2, 3 , and 4). Future improvements in the comparisons with theoretical models will need to take into account carefully such small systematic effects in the photometry.

\section{D.5. Procedures for Solar-Type Stars}

In the $V, J, H$, and $K$ bands, the colors of solar-type stars depart significantly from a Rayleigh-Jeans shape and we have to be careful in defining a sample of such stars. We determined average colors from the list of 36 solar-type stars in Table 12. The stellar parameters are from the Appalachian State Nstars Web site (Gray 2007), with a few extras from Soubiran \& Triaud (2004). A fit of temperature versus spectral type from the full Nstars database shows that the average G2V star is assigned a temperature of $5720 \mathrm{~K}$. The solar temperature of $5778 \mathrm{~K}$ is within the scatter, but either the Sun is relatively hot for its spectral class, or there is a systematic offset between its temperature and those assigned through the stellar models to other stars.

We used the Hipparcos photometry at $V$ to eliminate stars from the sample with $V-K_{S}$ colors differing by more than $0.1 \mathrm{mag}$ from expectations for their spectral types. These stars are systematically much closer than the A stars discussed above, so the color deviations are less likely to arise from reddening. In support of this conclusion, there are nearly as many blue as red deviations. We attribute the discordant values to errors in the spectral types.

Each $V-K_{S}$ was corrected to the equivalent value for $5778 \mathrm{~K}$ according to a relationship fitting the temperature of the star and the standard $V-K$ colors from Tokunaga (2000). This relationship was set to zero at $5778 \mathrm{~K}$, so it acts only to take out the variations with temperature but not to cause an overall shift in color. We ignored reddening because the maximum distance of the sample members, $40 \mathrm{pc}$, puts them within the Local Bubble of a very low density interstellar medium (e.g., Knude \& Hog 1998 and references therein).

Although we prefer to use the Hipparcos $V$ magnitudes because they are homogeneous over the entire sky, to do so we need to allow for possible systematic offsets relative to Johnson
Table 12

Properties of Solar Analog Stars

\begin{tabular}{|c|c|c|c|c|}
\hline Name & $T_{\text {eff }}$ & $\log (g)$ & $M / H$ & $V-K_{S}$ \\
\hline Sun & 5778 & 4.44 & 0 & 1.57 \\
\hline HD 001562 & 5756 & 4.43 & -0.22 & 1.54 \\
\hline HD 003821 & 5785 & 4.40 & -0.07 & 1.50 \\
\hline HD 008262 & 5861 & 4.34 & -0.13 & 1.57 \\
\hline HD 009986 & 5749 & 4.39 & -0.03 & 1.55 \\
\hline HD 010086 & 5659 & 4.61 & -0.02 & 1.62 \\
\hline HD 010145 & 5673 & 4.40 & -0.01 & 1.64 \\
\hline HD 011131 & 5819 & 4.53 & -0.02 & 1.57 \\
\hline HD 012846 & 5667 & 4.38 & -0.25 & 1.60 \\
\hline HD 020619 & 5666 & 4.50 & -0.25 & 1.58 \\
\hline HD 041330 & 5858 & 4.25 & -0.20 & 1.52 \\
\hline HD 042618 & 5714 & 4.58 & -0.16 & 1.55 \\
\hline HD 063433 & 5691 & 4.60 & -0.03 & 1.64 \\
\hline HD 071148 & 5756 & 4.35 & -0.02 & 1.49 \\
\hline HD 073350 & 5754 & 4.37 & 0.04 & 1.52 \\
\hline HD 075767 & 5741 & 4.42 & -0.08 & 1.57 \\
\hline HD 088072 & 5746 & 4.31 & 0.01 & 1.55 \\
\hline HD 089269 & 5674 & 4.40 & -0.23 & 1.65 \\
\hline HD 090508 & 5779 & 4.24 & -0.23 & 1.55 \\
\hline HD 114174 & 5750 & 4.34 & 0.07 & 1.58 \\
\hline HD 139777 & 5703 & 4.55 & -0.05 & 1.50 \\
\hline HD 142093 & 5859 & 4.55 & -0.15 & 1.49 \\
\hline HD 146946 & 5854 & 4.21 & -0.21 & 1.62 \\
\hline HD 147044 & 5849 & 4.29 & -0.03 & 1.45 \\
\hline HD 159222 & 5788 & 4.25 & 0.12 & 1.52 \\
\hline HD 165401 & 5798 & 4.27 & -0.29 & 1.55 \\
\hline HD 166435 & 5741 & 4.41 & -0.07 & 1.52 \\
\hline HD 168009 & 5801 & 4.09 & -0.04 & 1.54 \\
\hline HD 177082 & 5733 & 4.25 & -0.11 & 1.60 \\
\hline HD 186427 & 5753 & 4.25 & 0.06 & 1.60 \\
\hline HD 187237 & 5788 & 4.61 & 0.06 & 1.53 \\
\hline HD 196850 & 5792 & 4.30 & -0.12 & 1.53 \\
\hline HD 197076 & 5842 & 4.34 & -0.02 & 1.51 \\
\hline HD 202108 & 5665 & 4.38 & -0.30 & 1.57 \\
\hline HD 217813 & 5861 & 4.41 & -0.03 & 1.50 \\
\hline HD 218739 & 5788 & 4.41 & 0.06 & 1.44 \\
\hline HD 224465 & 5664 & 4.22 & -0.12 & 1.58 \\
\hline
\end{tabular}

$V$. These effects should be small for A stars because of their nearly zero colors. To evaluate them for solar-type stars, we used the sample of 102 stars listed in the Nstars database as being of G-type and for which there was high-quality $V$ photometry, to find a net offset of $\left\langle V_{\text {Hipp }}-V_{\text {Johnson }}\right\rangle=-0.007 \pm 0.002$. We corrected the measured $V-K_{S}$ color accordingly. We also found two stars in our solar-type sample, HD 139777 and HD 218739, with large offsets between the N stars tabulated ground-based magnitudes and those from Hipparcos (0.07$0.08 \mathrm{mag}$ ). Multiple ground-based measurements plus the $V-K$ color showed that the ground-based results were correct, and we substituted them. Otherwise, the standard deviation in the difference of Hipparcos and ground-based $V$ magnitude derived from $u v b y$ was only $1.6 \%$, confirming the high quality of the Hipparcos data (and of the ground-based $u v b y$ data also).

\section{APPENDIX E}

\section{CALCULATION OF EFFECTIVE, NOMINAL, AND ISOPHOTAL WAVELENGTHS}

Given $R(\lambda)$ as the relative response function of the photometric band, the equivalent band width is 


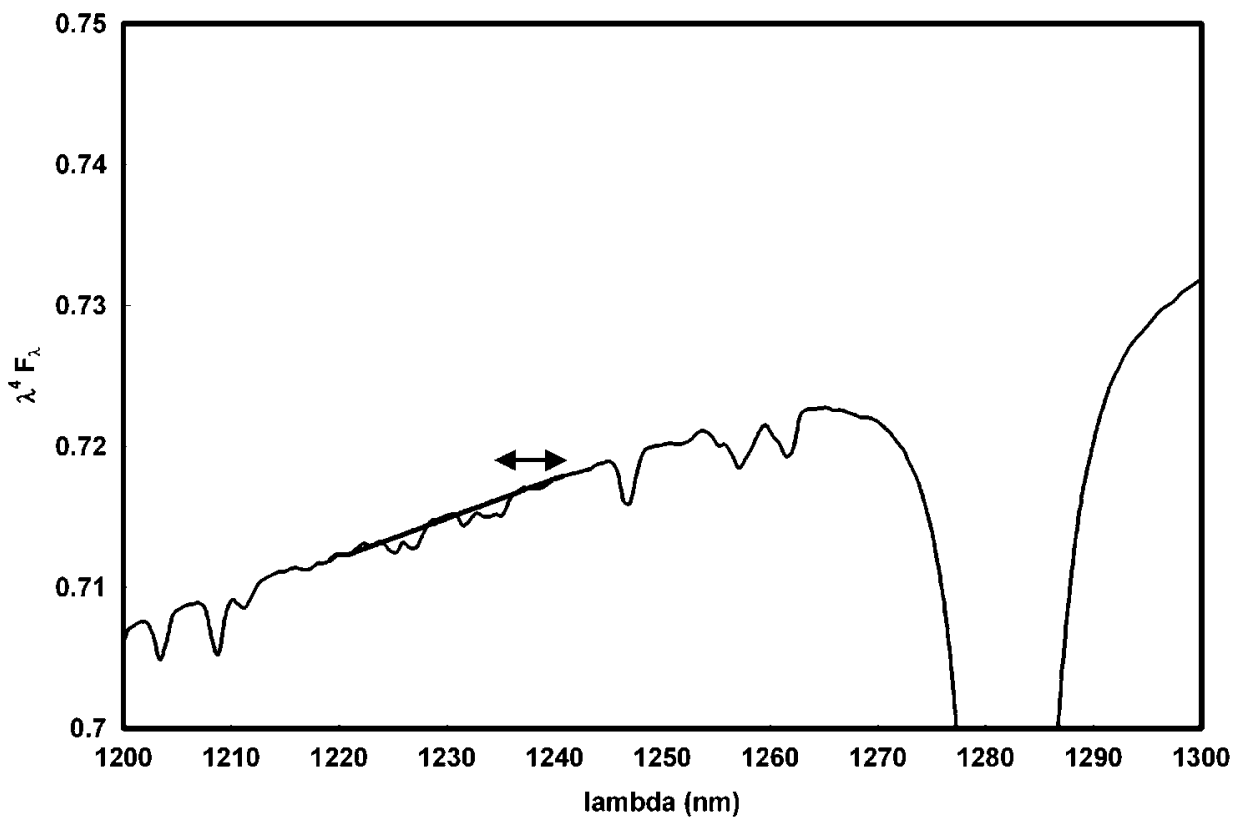

Figure 4. Interpolation over spectral absorptions in the $J$ band. The horizontal arrow shows the approximate range of the various band-defining wavelengths.

$$
\mathrm{BW}=\frac{1}{R_{\max }} \int R(\lambda) d \lambda
$$

where $R_{\max }$ is the maximum value of $R(\lambda)$ (often $R(\lambda)$ is normalized to 1$)$. The average flux in the band is

$$
\left\langle F_{\lambda}\right\rangle=\frac{\int \lambda F_{\lambda}(\lambda) R(\lambda) d \lambda}{\int \lambda R(\lambda) d \lambda},
$$

or equivalently is the total flux passed by the band divided by the band width.

To idealize measurements made through a given photometric band to a monochromatic equivalent flux, we need to assign a specific wavelength to the measurement. The mean, or effective, wavelength is defined by the following equation:

$$
\lambda_{0}=\frac{\int \lambda R(\lambda) d \lambda}{\int R(\lambda) d \lambda} .
$$

Examples are given in Table 6. The $\mathrm{ZP}$ is conventionally defined as the flux density of "Vega" at $\lambda_{0}$, but the stellar absorption features pose complications. The ZPs in Table 6 are determined by interpolating over the absorptions in the stellar spectrum and then averaging $\lambda^{4} F_{\lambda}$ over a $1 \%$ bandpass. Any measurement expressed in terms of the mean wavelength of the photometric band needs to be accompanied by a color correction term that converts the apparent flux density to the value at $\lambda_{0}$ for the specific source SED that reproduces the observed signal strength (e.g., Low \& Rieke 1974). The color correction is normalized to the result for a source with $F_{\lambda}(\lambda)=$ constant across the band. The absorption features in stellar spectra can make computation of a precise color correction problematical, but alternatively one can be based on a blackbody spectrum. We have taken this approach for the illustrative values in Table 6 . They are given in the sense that a flat spectrum that gives the same signal as a blackbody of the specified temperature will be brighter at the mean wavelength by the tabulated factor. It is convenient in many cases to set the color correction to zero for hot stars, which can be accomplished by an offset in the set of color terms with an appropriate counter-adjustment in the nominal ZP flux density (e.g., Low \& Rieke 1974; MIPS Handbook).

Another way to circumvent the effects of stellar absorptions is to use the "average ZP", $\left\langle F_{\lambda}\right\rangle$, where the flux density is that of "Vega." Where the stellar spectrum is smooth and follows a Planck curve closely, the average flux in the band is reproduced reasonably well by the stellar flux density at the mean wavelength times the color correction. Where the stellar spectrum deviates from Planckian behavior (e.g., in the $H$ band due to the many members of the Brackett series), there may be significant deviations from this relation. In general cases, the use of a fiducial wavelength and ZP imposes limits in the achievable accuracy; it may be required to carry out the relevant integrals of the relative response function convolved with the object spectra.

Under many conditions, the value of the required color correction can be minimized by modifying the wavelength associated with the band to the "nominal wavelength" (Reach et al. 2005), defined as

$$
\lambda_{0}^{\prime}=\frac{\int \lambda^{2} R(\lambda) d \lambda}{\int \lambda R(\lambda) d \lambda} .
$$

Table 6 includes the ZP flux densities appropriate to this definition. Color corrections under this definition are relative to those for a source with $F_{\lambda}(\lambda) \propto \lambda$. Otherwise, the procedures and pitfalls are similar to those for the mean wavelength.

The definitions in Equations (3) and (4) have the advantages that they are simple in concept and that the wavelength associated with a band is independent of the source properties. Yet another definition is the isophotal wavelength, defined by

$$
F_{\lambda}\left(\lambda_{\text {iso }}\right)=\left\langle F_{\lambda} .\right\rangle \text {. }
$$

This approach has the advantage of subsuming the correction terms into the wavelength specification, providing a more 


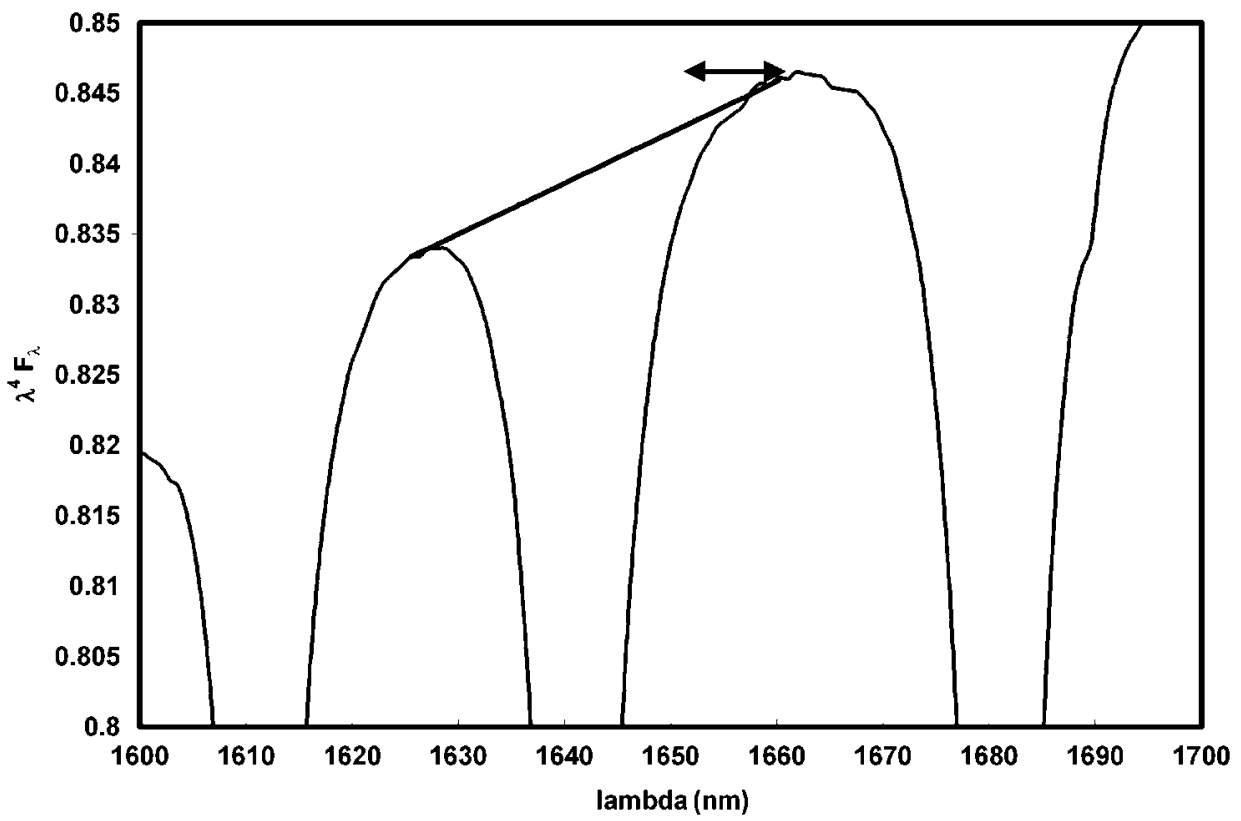

Figure 5. Interpolation over spectral absorptions in the $H$ band. The horizontal arrow shows the approximate range of the various band-defining wavelengths.

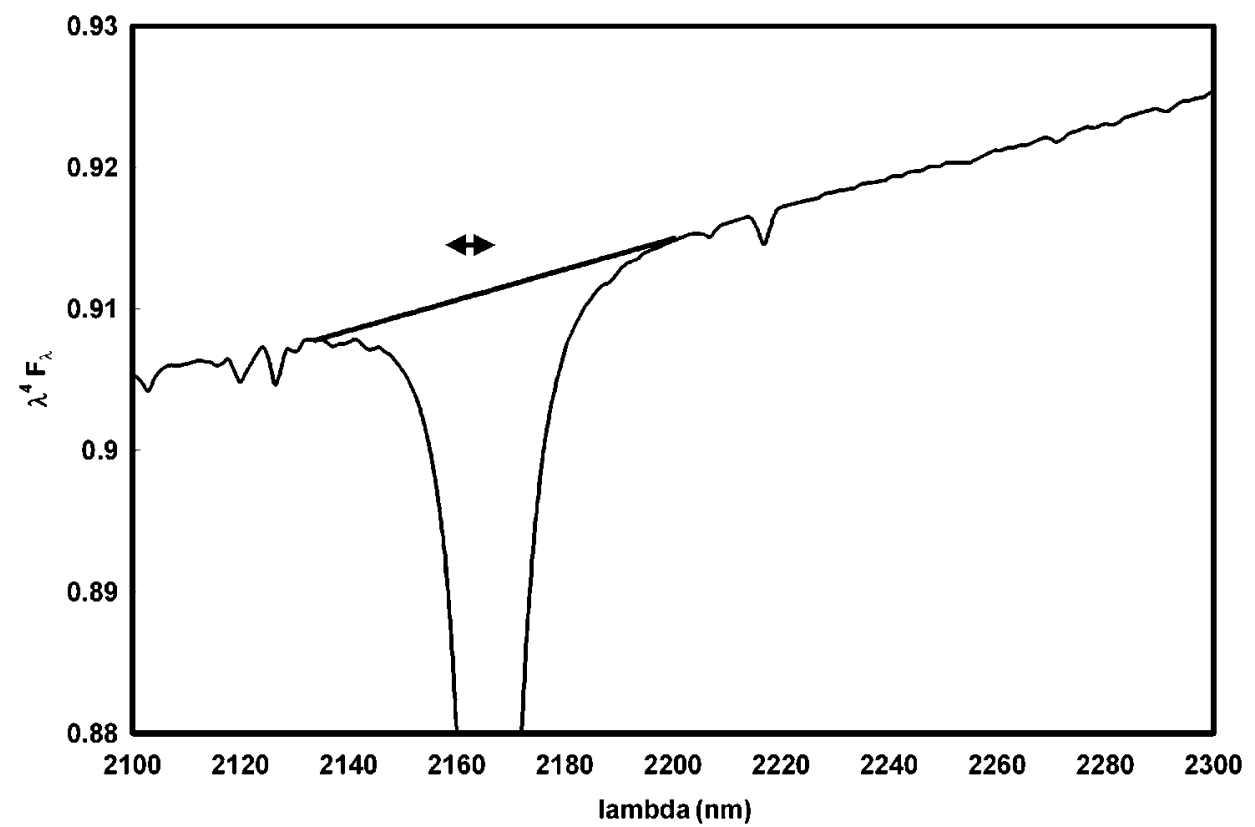

Figure 6. Interpolation over spectral absorptions in the $K_{S}$ band. The horizontal arrow shows the approximate range of the various band-defining wavelengths.

streamlined description of a measurement. However, it is potentially confusing to have different wavelengths associated with measurements of different sources in the identical photometric band, and relating measurements to the identical wavelength requires introduction of color corrections similar to those needed with the definitions in Equations (3) and (4) (Tokunaga \& Vacca 2005).

The issue of stellar features is complex in the use of $\lambda_{\text {iso }}$, where the basic definitions can be misleading (e.g., $F_{\lambda}\left(\lambda_{\text {iso }}\right)$ may depend on the resolution used to measure the spectrum of the source). To avoid this difficulty, one must use a continuum model of the stellar spectrum or interpolate over its spectral features. Golay (1974) suggests avoiding these difficulties by replacing the stellar spectrum with a suitable Planck function. Instead, for the 2MASS bands we have interpolated over any lines close to the fiducial wavelength (a procedure similar to that used by Cohen et al. 1992, 2003). We find that reliable interpolations can be based on $\lambda^{4} F_{\lambda}$. Since the IR is close to the Rayleigh-Jeans realm for Vega, the resulting function varies slowly and smoothly with wavelength, making it appropriate to use linear interpolations across line-contaminated regions. The interpolated values were converted to flux by dividing by $\lambda^{4}$. Figures 4-6 show our interpolations on the model spectrum of Vega. Table 13 lists the beginning and end wavelengths for the interpolations. Our calculated values of $\lambda_{\text {iso }}$ in Table 6 agree closely with those of Cohen et al. (2003). Therefore, many 
Table 13

Beginning and End Wavelengths for Isophotal Interpolations

\begin{tabular}{lcc}
\hline \hline & Beginning & End \\
\hline$J$ & $1.221 \mu \mathrm{m}$ & $1.240 \mu \mathrm{m}$ \\
$H$ & $1.6255 \mu \mathrm{m}$ & $1.660 \mu \mathrm{m}$ \\
$K_{S}$ & $2.134 \mu \mathrm{m}$ & $2.200 \mu \mathrm{m}$
\end{tabular}

aspects of their analysis, such as the water vapor dependence of the nominal bands and fiducial wavelengths, can be applied to our proposed calibration without change.

For the mid-IR space missions, color corrections as a function of source spectral characteristics can be found in the appropriate user handbooks. The MIPS calibration is for a mean wavelength, while the IRAC and IRAS ones are for a nominal wavelength. Because these corrections are tracked as official project values, we quote our results in a form that assumes they will be used as tabulated.

\section{REFERENCES}

Absil, O., et al. 2006, A\&A, 452, 237

Anders, E., \& Grevesse, N. 1989, Geochimica et Cosmochimica Acta, 53, 197

Aufdenberg, J. P., et al. 2006, ApJ, 645, 664

Aumann, H. H., et al. 1984, ApJL, 278, 23

Ayres, T. R., Plymate, C., \& Keller, C. U. 2006, ApJS, 165, 618

Becklin, E. E., Hansen, O., Kieffer, H., \& Neugebauer, G. 1973, AJ, 78, 1063

Beichman, C. A., Neugebauer, G., Habing, H. J., Clegg, P. E., \& Chester, T. J. 1988, IRAS Explanatory Supplement (Washington, DC: NASA) Section VI. C.2.a

Bell, K. L., \& Berrington, K. A. 1987, J. Phys. B: At. Mol. Phys., 20, 801

Bessell, M. S. 2005, ARAA, 43, 293

Bessell, M. S., \& Brett, J. M. 1988, PASP, 100, 1134

Bessell, M. S., Castelli, F., \& Plez, B. 1998, A\&A, 333, 231

Blackwell, D. E., Leggett, S. K., Petford, A. D., Mountain, C. M., \& Selby, M. J. 1983, MNRAS, 205, 897

Bohlin, R. C. 2007, The Future of Photometric Systems, PASP, 364, 315

Bohlin, R. C., Dickinson, M. E., \& Calzetti, D. 2001, AJ, 122, 2118

Bohlin, R. C., \& Gilliland, R. L. 2004, AJ, 127, 3508

Booth, A. J., Selby, M. J., Blackwell, D. E., Petford, A. D., \& Arribas, S. 1989, A\&A, 218, 167

Bouchet, P., Schmider, F. X., \& Manfroid, J. 1991, A\&AS, 91, 409

Campins, H., Rieke, G. H., \& Lebofsky, M. J. 1985, AJ, 90, 896

Carpenter, J. M. 2001, AJ, 121, 2851

Carter, B. S. 1990, MNRAS, 242, 1

Casagrande, L., Portinari, L., \& Flynn, C. 2006, MNRAS, 373, 13

Cayrel de Strobel, G. 1996, A\&A Rev., 7, 243

Ciardi, D. R., van Belle, G. T., Akeson, R. L., Thompson, R. R., Lada, E. A., \& Howell, S. B. 2001, ApJ, 559, 1147

Cohen, M., Walker, R. G., Barlow, M. J., \& Deacon, J. R. 1992, AJ, 104, 1650

Cohen, M., Wheaton, W. A., \& Megeath, S. T. 2003, AJ, 126, 1090

Colina, L., Bohlin, R. C., \& Castelli, F. 1996, AJ, 112, 307

Cutri, R. M., et al. 2003, The IRSA 2MASS All-Sky Point Source Catalog (Pasadena: NASA/IPAC)

Decin, L. 2000, PhD Thesis, Univ. of Leuven, Belgium

Decin, L., Vandenbussche, B., Waelkens, C., Eriksson, K., Gustafsson, B., Plez, B., Sauval, A. J., \& Hinkle, K. 2003, A\&A, 400, 679

Elias, J. H., Frogel, J. A., \& Humphreys, R. M. 1985, ApJS, 57, 91

Elias, J. H., Frogel, J. A., Matthews, K., \& Neugebauer, G. 1982, AJ, 87, 1092
Engelbracht, C., et al. 2007, PASP, 119, 994

Engelke, C. W. 1992, AJ, 104, 1248

Fazio, G. G., et al. 2004, ApJS, 154, 39

Fontenla, J. M., Avrett, E., Thuillier, G., \& Harder, J. 2006, ApJ, 639, 441

Golay, M. 1974, Introduction to Stellar Photometry (Dordrecht: Reidel), 40

Gray, R. O. 1998, PASP, 116, 482

Gray, R. O. 2007, http://stellar.phys.appstate.edu/

Gulliver, A. F., Hill, G., \& Adelman, S. J. 1994, ApJL, 429, L81

Hammersley, P. L., Jourdain de Muizon, M., Kessler, M. F., Bouchet, P., Joseph,

R. D., Habing, H. J., Salama, A., \& Metcalfe, L. 1998, A\&AS, 128, 307

Harris, M. J., Lambert, D. L., \& Goldman, A. 1987, MNRAS, 224, 237

Holmberg, J., Flynn, C., \& Portinari, L. 2006, MNRAS, 367, 449

Holweger, H., \& Müller, E. A. 1974, Solar Phys., 39, 19

Johnson, H. L. 1965a, Comm. Lunar Plan. Lab., 3, 73

Johnson, H. L. 1965b, ApJ, 141, 923

Johnson, H. L., Iriarte, B., Mitchell, R. I., \& Wisniewski, W. Z. 1966, Comm. Lunar Plan. Lab., 4, 99

Johnson, H. L., MacArthur, J. W., \& Mitchell, R. I. 1968, ApJ, 152, 465

Johnson, H. L., \& Morgan, W. W. 1953, ApJ, 117, 313

Kidger, M. R., \& Martín-Luis, F. 2003, AJ, 125, 3311

Knude, J., \& Hog, E. 1998, A\&A, 338, 897

Koornneef, J. 1983, A\&AS, 51, 489

Kurucz, R. L. 2005, http://kurucz.harvard.edu

Labs, D., \& Neckel, H. 1968, Z. Astrophys., 69, 1

Labs, D., \& Neckel, H. 1970, Solar Phys., 15, 79

Lee, T. A. 1968 , ApJ, 152, 913

Low, F. J., \& Rieke, G. H. 1974, in Methods of Experimental Physics, Vol. 12, Part A, ed. N. Carleton (New York: Academic)

Maltby, P., Avrett, E. H., Carlsson, M., Kjeldseth-Moe, O., Kurucz, R. L., \& Loesser, R. 1986, ApJ, 306, 284

Mégessier, C. 1995, A\&A, 296, 771

MIPS Data Handbook: http://ssc.spitzer.caltech.edu/mips/dh

Mountain, C. M., Selby, M. J., Leggett, S. K., Blackwell, D. E., \& Petford, A. D. 1985, A\&A, 151, 399

NASA/IPAC Infrared Science Archive 2007, http://irsa.ipac.caltech.edu/data/ SPITZER/FEPS/

Perryman, M. A. C., et al. 1997, A\&A, 323, L49

Plez, B., Smith, V. V., \& Lambert, D. L. 1993, ApJ, 418, 812

Price, S. D. 2004, Space Sci. Rev., 113, 409

Price, S. D., Paxson, C., Engelke, C., \& Murdock, T. L. 2004, AJ, 128, 889

Reach, W. T., et al. 2005, PASP, 117, 978

Rieke, G. H., \& Lebofsky, M. J. 1985, ApJ, 288, 618

Rieke, G. H., Lebofsky, M. J., \& Low, F. J. 1985, AJ, 90, 900

Rieke, G. H., et al. 2004, ApJS, 154, 25

Rieke, G. H., et al. 2005, ApJ, 620, 1010

Saiedy, F. 1960, MNRAS, 121, 483

Saiedy, F., \& Goody, R. M. 1959, MNRAS, 119, 213

Selby, M. J., Mountain, C. M., Blackwell, D. E., Petford, A. D., \& Leggett, S. K. 1983, MNRAS, 203, 795

Soubiran, C., \& Triaud, A. 2004, A\&A, 418, 1089

Spitzer Science Center 2007, http://ssc.spitzer.caltech.edu/postbcd/spice.html/

Su, K. Y. L., et al. 2006, ApJ, 653, 675

Thuillier, G., Hersé, M., Labs, D., Foujols, T., Peetermans, W., Gillotay, D., Simon, P. C., \& Mandel, H. 2003, Solar Phys., 214, 1

Tokunaga, A. T. 2000, in Allen's Astrophysical Quantities, ed. A. N. Cox (4th ed.; New York: Springer), 143

Tokunaga, A. T., \& Vacca, W. D. 2005, PASP, 117, 421

Vernazza, J. E., Avrett, E. H., \& Loeser, R. 1976, ApJS, 30, 1

Walker, R. G. 1969, Phil. Trans. Roy. Soc. A, 264, 209

Wallace, L., \& Livingston, W. 2003, National Solar Obsevatory Technical Report \#03-001

Wright, J. T., Marcy, G. W., Butler, R. P., \& Vogt, S. S. 2004, ApJS, 152,261 